\title{
Modular Self-Reconfigurable Robotic Systems: A Survey on Hardware Architectures
}

\section{S. Sankhar Reddy Chennareddy, Anita Agrawal, and Anupama Karuppiah}

EEE Department, NH-17B, BITS Pilani KK Birla Goa Campus, Goa 403726, India

Correspondence should be addressed to S. Sankhar Reddy Chennareddy; sankhar@goa.bits-pilani.ac.in

Received 22 November 2016; Accepted 26 February 2017; Published 15 March 2017

Academic Editor: Shahram Payandeh

Copyright ( 2017 S. Sankhar Reddy Chennareddy et al. This is an open access article distributed under the Creative Commons Attribution License, which permits unrestricted use, distribution, and reproduction in any medium, provided the original work is properly cited.

\begin{abstract}
Modular self-reconfigurable robots present wide and unique solutions for growing demands in the domains of space exploration, automation, consumer products, and so forth. The higher utilization factor and self-healing capabilities are most demanded traits in robotics for real world applications and modular robotics offer better solutions in these perspectives in relation to traditional robotics. The researchers in robotics domain identified various applications and prototyped numerous robotic models while addressing constraints such as homogeneity, reconfigurability, form factor, and power consumption. The diversified nature of various modular robotic solutions proposed for real world applications and utilization of different sensor and actuator interfacing techniques along with physical model optimizations presents implicit challenges to researchers while identifying and visualizing the merits/demerits of various approaches to a solution. This paper attempts to simplify the comparison of various hardware prototypes by providing a brief study on hardware architectures of modular robots capable of self-healing and reconfiguration along with design techniques adopted in modeling robots, interfacing technologies, and so forth over the past 25 years.
\end{abstract}

\section{Introduction}

Modular robotics provides a unique advantage over traditional robotic technologies in terms of reconfigurability, reusability, and ease in manufacturing. The traditional robotic designs such as robotic arms and hexapods. provide unique solutions to each real world application and the generated prototypes are generally inflexible for the rest of the applications. Most of the traditional robotic solutions are operated in a controlled environment and any changes in environments often make these traditional solutions inflexible due to lack of their adaptive nature. The repair and maintenance of such conventional designs generally require separate trained personnel for each model and hence increasing the average resource consumption in industries. The next phase of robotic designs is developed in the perspective of assembly of modular units for increasing ease of repairing, replacing, control, and so forth. The researchers in later phases of development introduced the concept of automation, selfhealing, reconfiguration, and so forth creating a modular self-reconfigurable robots (MSRR). Many applications such as management of large facilities [1], space exploration [2], surveillance in military zones, disaster management, and prosthetics for physically disabled often require adaptable and self-healing abilities and MSRR is often considered as a viable solution for the same. The major difference of MSRR designs over modular robotic designs can be visualized as the abilities of designs to attach/detach in/from a formation as per the requirement of application with minimal human intervention.

The growing demand for reusable, space constrained, and multipurpose solutions for real world applications is a great motivator for research in the field of MSRR. The researchers in domain of MSRR provided numerous solutions via various prototype designs, communication algorithms, coordination, and dispersion techniques using selected test scenarios. The development of novel prototypes for MSRR is an analytical process that often has deep roots in intuition and derives better fruits from experience on basic locomotion and laws of physics. Majority of the robotic modules developed so far utilized limited resources available at time of development and have restricted capabilities due to slow technological 
advancements in the areas of sensors, hardware prototyping, actuators, communication interfaces, and so forth. The different approaches adopted by researchers to validate the designs and prototypes make relative comparison between the robotic modules a fairly difficult process and present challenges in quantifying and understanding merits/demerits of various designs.

A detailed survey on wide range of solutions for modular designs of outer structures, physical interfaces between modules, communication protocols, sensor technologies for docking and alignment, coordinate movement algorithms, environment characteristics, and so forth provides better understanding to novel researchers about merits and demerits of previous designs so that better solutions can be provided with the utilization of latest technologies. This paper scope is limited to study and summarize the hardware architectures along with sensor and interfacing technologies of various MSRR.

\section{Modular Robots, Hardware Architectures}

The hardware architectures in MSRR are evolving along with the technologies and so does the paradigm used for categorizing the robots. The first prototype developed in MSRR research is CEBOT consisting of heterogeneous separate units capable of binding together and since then the research was directed to development of systems capable for forming different structures mimicking biological organisms. Yim et al. [2, 3] suggested two classifications of modular robotic systems: classification based on structures formed by MSRR and classification based on reconfiguration strategies. Gilpin and Rus [4] added few more subclassifications under structures category by including research from microelectronic mechanical systems (MEMS) and other latest developments in MSRR by the time of publication. Moubarak and Ben-Tzvi [5] categorized MSRR systems based on locomotion of the individual modules and coordinated structures along with form factors. The classifications proposed so far are categorized as per the state-of-the-art research in recent MSRR technologies, prototypes, and so forth available till the data of publication. The recent research in MSRR is generating solutions that are falling in the middle of earlier classifications and the identification of a category and subcategory for MSRR robots is becoming difficult due to recent sophisticated designs and features of robots.

The classification of MSRR based on various categories and subcategories such as physical characteristics, abilities, and so forth is provided in Figure 1. The widely accepted classification is in the perspective of possible structural formations when the independent MSRR are brought together and five subcategories are recognized under structures as per the current MSRR research: lattice, chain, hybrid, truss, and free-form.

The MSRR designed for lattice structures are inspired from atomic structures like cubic centered lattice, tetrahedron, and so forth and are equipped with actuators to form similar structures. The individual robotic units occupy discrete positions in space and lack capabilities to reach random positions/orientations if necessary due to limitation in actuator assemblies. The lattice architectures provide easy control mechanisms and do not often require closed loop control due to their defined actuator positions in $2 \mathrm{D}$ and $3 \mathrm{D}$ space. The robotic units under chain category are generally serially connected robotic units and are capable of forming complex structures like snakes, centipedes, and so forth. The actuators of these robots are assembled to provide end effectors random positions in space. The control of chained systems is more complex and often requires feedback to confirm the position of modules in space for reconfiguring structures. The hybrid designs provide more advantages compared to lattice and chain robotic structures due to their capabilities in easy adaptation to surroundings by forming both lattice, chained and mixture of both. The MSRR with truss based designs support formation of random structures due to the employment of telescopic links and heterogeneous units for forming structures but require complex algorithms for handling assembly and formation of structures. The freeform category MSRR are generally more flexible in the perspective of attaching and detaching from the system. They can form arbitrary structures and normally maintain weak bonds between the neighbors. The chain and hybrid differ from the free-form structures generally in terms of rigidity in bonding.

The sophisticated locomotion capabilities of MSRR are resultant of coordinated actions of many individual units aggregated in various structures. The capabilities (autonomous/semiautonomous/manual) of aggregation in MSRR for facilitating complex movements by reconfiguration rely on the actuator-sensor assemblies embedded in individual robotic designs. The MSRR designs equipped with wheels are capable of forming lattice or chain structures depending on the design and hence can be placed in mobile subcategory under locomotion in Figure 1. The majority of lattice and chain systems are designed without wheels on individual units and hence mobility is realized only by employment of coordination of robots. The aggregation of individual units requires human intervention to a certain degree for nonwheeled systems. These MSRR designs can be placed in coordinated subcategory under locomotion category shown in Figure 1. The external subcategory under locomotion in Figure 1 refers to the MSRR designs that rely on environmental stimulus/disturbances for locomotion as well as reconfiguration.

The recent contributions to MSRR attempted the concept of employing disturbances and vibrations in environment for assembly of robots introducing along with addressing uncertainty in reconfiguration structures and hence creating two subcategories based on reconfiguration: deterministic and stochastic. The deterministic reconfiguration type of MSRR has precise control over the structures, assembly, and reconfiguration by employing either closed loop control or advanced actuator assemblies. The stochastic type of MSRR mostly does not have control over the assembly of units but generally retains the ability for disassembly. Hence the reconfiguration after completing a particular structure assembly and the time required for the same has major contribution from environmental factors.

Many researchers developed designs in Micro to Macro sizes for addressing various scenarios in MSRR. The form 


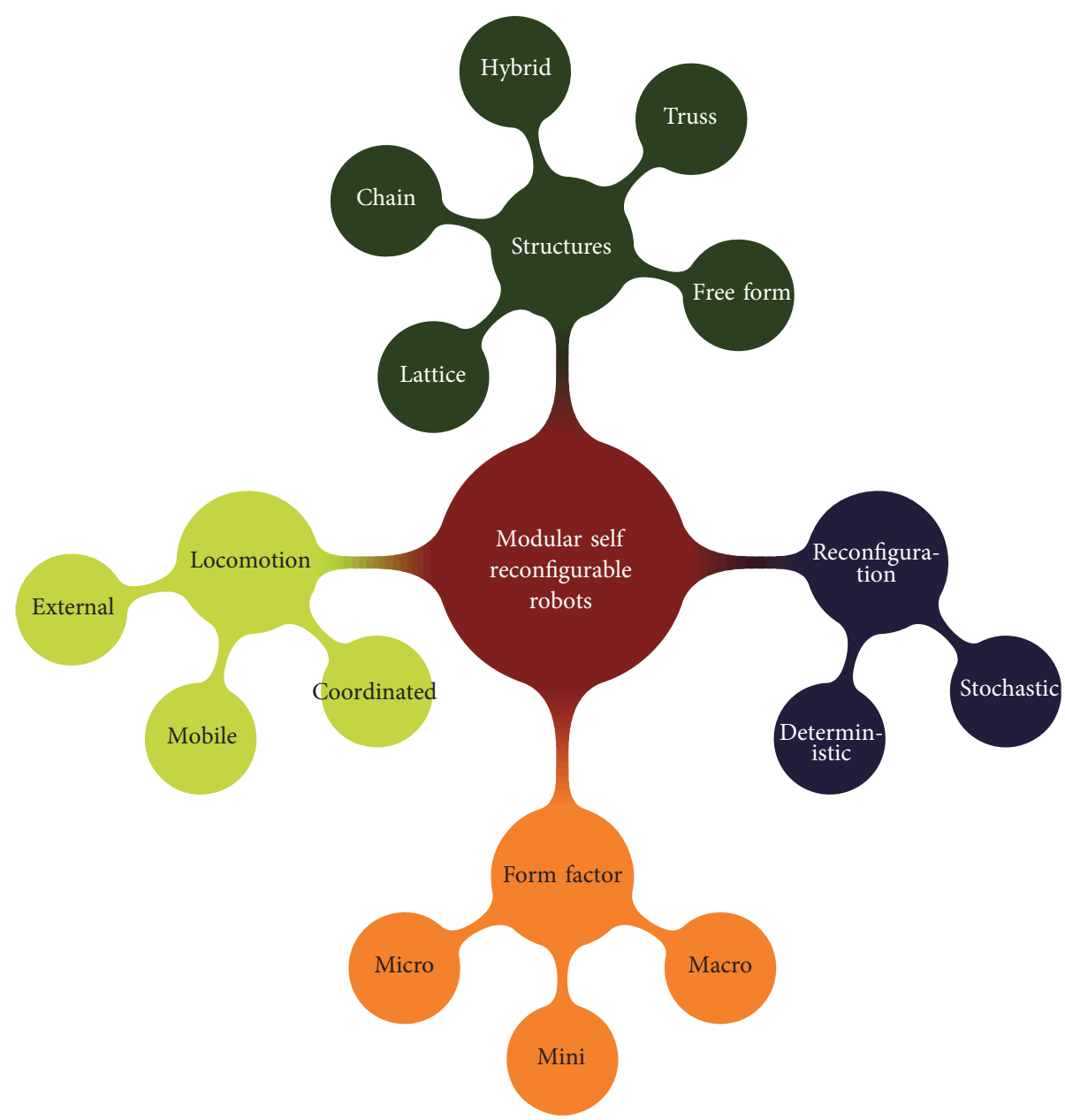

FIGURE 1: Classification of MSRR designs based on hardware characteristics.

factor scaling is completed at a trade-off with capabilities and also increased dependency on events happening in the surrounding environment. Henceforth in this paper, the MSRR robots occupying volume equal to and more than a cube of $5 \mathrm{~cm}$ side are referred to as macro structures, models occupying less than volume of macro designs but visible to naked eye are referred to as mini structures, and designs not easily visible to naked eye are referred to as micro structures. In this paper we adopted the widely accepted classification of MSRR-classification based on structures for broadly summarizing the research so far. The other categories are related implicitly while providing the details of locomotion, dimension, and mobility.

2.1. Lattice Structured Systems. The metamorphic robotic system [6-8] is the first lattice structure category robotic design capable of changing structures in $2 \mathrm{D}$ environment. The authors explored the idea of hexagonal and square lattice structures using the metamorphic robotics systems. A hexagonal skeleton was developed for mimicking the robot outer structure with 6 servo motors at each corner and male and female connectors on alternate sides for docking as shown in Figure 2(a). After successful docking between the cells, each cell can revolve around the periphery of neighboring cell by gradually changing their structure. The square structured prototypes for lattice structures employ sliding mechanism using gender based connectivity for movement along the lattice structures.

Murata et al. developed a 2D lattice category MSRR called Fracta [9]. The individual robot in Fracta consists of a top, a bottom module with permanent magnets, and a middle module equipped with electromagnets. The assembly is shown in Figure 2(b). The docking process begins with insertion of middle layer into the empty space between the top and bottom layer of neighboring modules by activating electromagnets. The operating principle was tested using modules equipped with castors on frictional less surface.

Molecule [10] is a 3D structure supporting design developed by Rus D. and each unit consists of two atoms and a right angle rigid bond binding them. The connectors equipped with electromagnets are present on side faces of each atom. The bonded two-atom system is referred to as "Molecule" and each atom has two degrees of freedom (DOF) with one provided by motor at a connector on face and another due to 


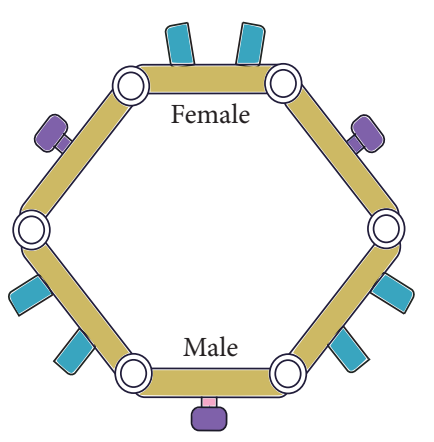

(a)

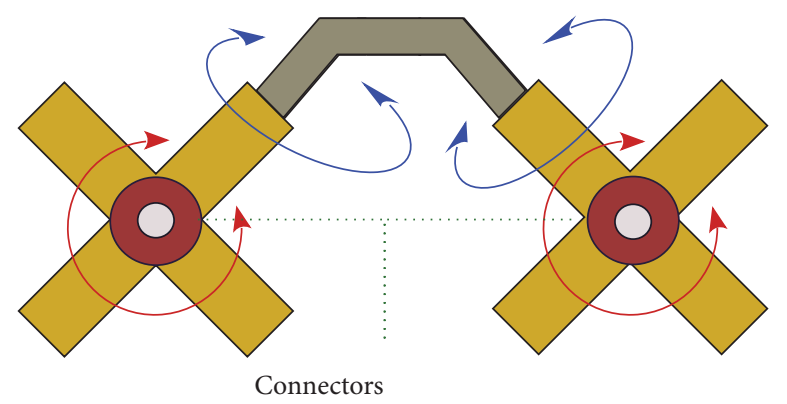

Side view

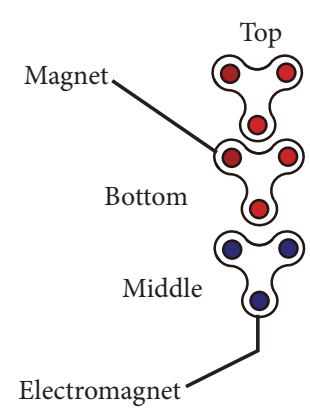

(b)

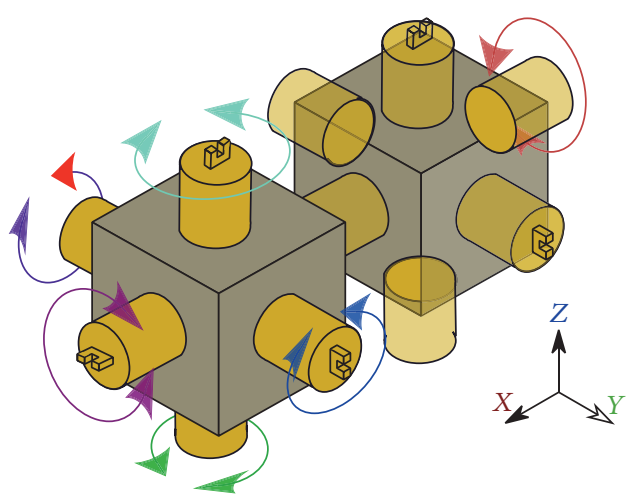

(d)

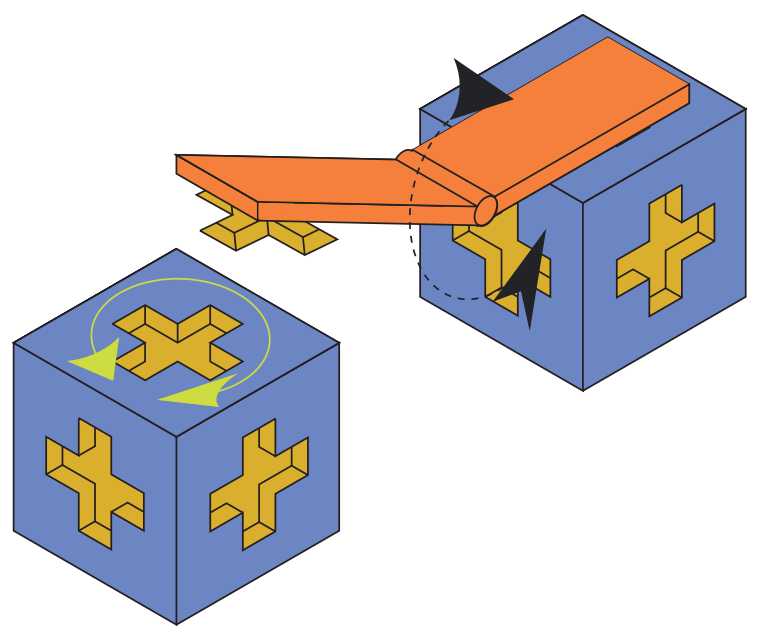

(e)

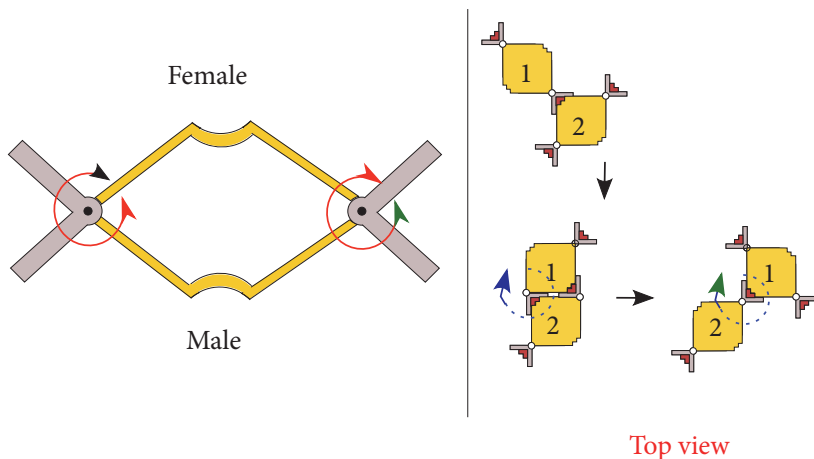

(f)

FIgURE 2: Lattice MSRR hardware models: (a) metamorphic, (b) Fracta, (c) molecule, (d) 3D unit, (e) I-cubes, and (f) Microunit 1.

motor at the bond as shown in Figure 2(c). The Molecule as a whole provides $4 \mathrm{DOF}$ and can be used for creating arbitrary structures like walls.

Kurokawa et al. prototyped a 3D unit [11, 12] in cubical structure with connectors on all faces. Each connector can rotate independently along their axis providing the $3 \mathrm{D}$ unit 6 DOF as shown in Figure 2(d). The connectors on all faces are connected to a single $7 \mathrm{~W}$ motor using worm gear mechanism controlled by independent solenoid driven switching technique for each arm. The arms are connected using connection cuff capable of moving back and forth along the axis of arm. The connection hand mounted on cuff closes at one extreme of sliding displacement and opens at the other.

The I-Cubes proposed by Ünsal et al. in $[13,14]$ is another cubical structure robotic design with two units: cubes and links. The faces of cubes have female connectors to mount the links using lock and key mechanism. A cube at a given time can have zero to six links connected to its faces. The links are independently controlled multijoint unit shared and transferred between cubes. The horizontal beam of link constitutes a joint at the center of two horizontal beams and can be rotated as shown in Figure 2(e). The cubes can 
rotate with respect to link after successful latching and hence providing locomotion to the cubes present in the system.

A mini form factor design referred to as Microunit [15, 16] further is developed by Yoshida et al. Microunit was prototyped in two different models and each module in the system has square skeleton structure with two static female connecting parts at two ends of a diagonal and rotating male connecting parts at the end of other diagonal as shown in Figure 2(f). The first prototype designed can form structures in $2 \mathrm{D}$ with docking controlled by torsion springs made from shape memory alloys (SMA). The design employs torsion springs and stoppers coupled with SMA for generating rotation mechanism. The authors also attempted further miniaturization of modules by removing the control unit present in earlier prototype and designed second model providing capabilities for forming structures in 3D.

The vertical robot published in [17] is a cubical structure of $90 \mathrm{~mm}$ side independent units. Each cube is equipped with two hands each lying on parallel side faces similar to human hands and the rest of the faces are equipped with magnetic sheets. The cells are capable of extending hands and rotation of the same only along the axis normal to surface they are mounted on. The design facilitates movement of robots only along vertical plane and hence stacking is the only method supported for navigation. The hands of two robots can be docked for lifting and the docking technique is facilitated by a genderless lock and key passive connector. The extension of hands is controlled using sliding mechanism.

Crystalline [18] is a cuboid structured robot with expansion and retraction capabilities on side faces. The expansion and retraction of faces are performed on all sides simultaneously using rack and pinion mechanism. The active connection mechanism is present on two neighboring side faces and passive connector mechanism is present on others. Since the system is not designed to docking on top and bottom faces, the crystalline MSRR structures are limited to $2 \mathrm{D}$ scenarios. The telecubes module [19] developed by Suh et al. is an improvisation to crystalline design with support for 3D structures. The six faces on each module can expand and contract in the direction normal to face similar to crystalline. Unlike crystalline, telecubes can move in vertical axis and hence has capabilities of forming 3D structures. Each face on telecubes module is divided into four quadrants with magnet pole pieces in odd and magnetic metal in even quadrants with chamfered borders for passive docking. The modules couple when they are close to each other since the connection plates on them are mirror images and the SMA springs present in the system pull magnetic pole pieces inside for undocking. The cubic structured module, EM-cubes published in [20], also employed magnets on four faces for docking and locomotion. The permanent magnets installed provide firm bonding and electromagnets facilitate locomotion. The electromagnets are activated alternatively to create attractive and repulsive forces simultaneously generating couple force at two ends of cube for locomotion.

M-blocks developed by Romanishin et al. are cubical MSRR prototyped in two versions: M-blocks [21] and 3D Mblocks [22]. The M-blocks and 3D M-blocks are equipped with an inertial actuator at center of body for applying

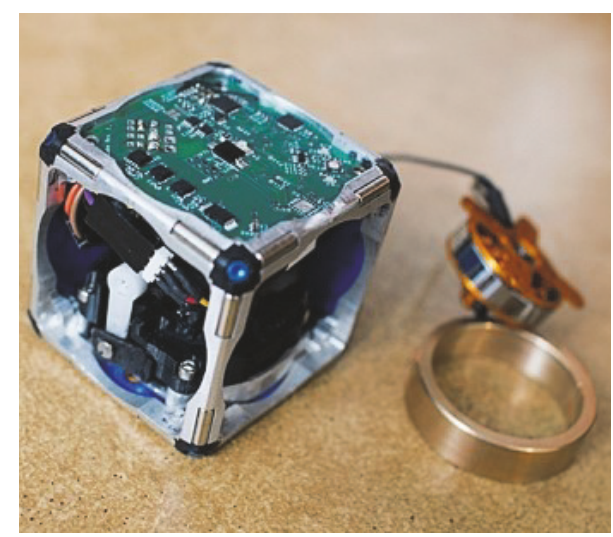

FIGURE 3: M-block MSRR (picture courtesy of M. Scott Brauer, source: http://news.mit.edu/2013/simple-scheme-for-self-assembling-robots-1004).

controlled torque at center of mass of the module and hence rotating the M-block MSRR in clockwise and anticlockwise directions. The M-block cells have capabilities of individual movement for docking and coordinate movement. The faces and edges of both models are embedded with permanent magnets as shown in Figure 3. The rapidly accelerating and decelerating internal rotation mechanism sources the locomotion and edge magnets control locomotion of modules around other robots using pivot action. The face magnets support alignment between the modules after locomotion. The M-blocks provide actuation in single direction and $3 \mathrm{D}$ M-blocks can actuate in six directions by changing inertial actuator orientation to any of three orthogonal axes for 3D movements. A mini form factor MSRR, MICHE [23], is designed for forming lattice structures in 3D with the aid of environment. Three faces of cubic structured MICHE are equipped with switchable magnets and the rest of the faces are covered with steel plates. The magnets are placed away from geometric center of plates for avoiding repulsion forces between magnets of two robots during docking. The magnet switching is controlled by internal microcontrollers communicating via IR transceivers and hence providing capabilities for retaining structures to MICHE MSRR. The MICHE MSRR falls under stochastic category for its dependence on environment for aggregation and locomotion. Pebbles [24] is another stochastic category cubic structure designed to form lattice structures in 2D. The four side faces on the robot can act as a connection plates due to their internal contact with four custom designed electropermanent magnets.

White et al. proposed stochastic robotic modules prototyped in two models with one supporting only $2 \mathrm{D}$ structures [25] and another supporting 3D structures [26]. The 2D structure modules are designed in both triangular and square base structures. The sides of a module's base are equipped with electromagnet for coupling. The docking and undocking are controlled by actuation and deactuation of electromagnets enabled via $\mathrm{H}$-bridges. The stochastic $3 \mathrm{D}$ version modules are cubic structures of $10 \mathrm{~cm}$ side with permanent magnets placed radially from center and electromagnets at the center 
of each face. The latching/unlatching is controlled by polarity of the electromagnet. The Programmable Parts [27] MSRR is another stochastic category robot with a triangular chassis equipped with latching mechanism on all sides. Each side is equipped with a fixed magnet and a rotating magnet controlled by DC motor placed adjacent to each other. During latching process, the fixed magnets of a module face rotating magnets of other robotic modules. Hence the modules can undock by retracting magnets by rotation in the self- and neighboring module. The IR sensors inserted into sides handle communication between the modules. The X-BOT [28, 29] MSRR consists of " $X$ " shaped cuboid modules capable of forming $2 \mathrm{D}$ structures stochastically. Each leg in " $\mathrm{X}$ " shape is equipped with a pair of compliant arms with magnets at their tips as shown in Figure 4. The arms bond different modules together and the coupling/decoupling process is controlled by push-pull process regulated by SMA wires wounded around the frame and arms.

The ATRON module proposed in $[30,31]$ is a lattice structured design along with minimal flexibility for forming chain structures in 3D as shown in Figure 5. The modules are composed of two hemispheres mounted upon each other on flat side and each hemisphere is capable of rotating $180^{\circ}$ independently. The two hooks (active male) and two passive female connectors placed equidistantly around periphery of each hemisphere in alternate positions facilitate docking. The hooks are driven by worm gears and female connectors are two rigid bars firmly connected to chassis of the module. The rotation of a hemisphere with respect to another provides locomotion in the structures.

The tetrapod structured PetRo MSRR [32] developed by Salem et al. is a self-mobile lattice category design proposed for forming 3D structures. The central hub and four legs together constitute a single unit in tetrapod shape. Each free end of legs is connected to wheels providing one DOF along leg axis and another DOF is added at the central hub perpendicular to leg axis with a rotation of $\pm 45^{\circ}$. The wheels are also proposed to play a role in connection plate between various PetRo modules forming complex structures similar to pets. The IR sensors present on the connector faces aids in alignment for docking. The grooved pins and chamfered holes on connection surfaces come opposite to each other after alignment and rotation and along with support from magnets the docking is completed successfully.

\subsection{Chain Structured Systems. CEBOT [33, 34] MSRR} belongs to mobile category comprising heterogeneous modules and has two hardware prototypes referred to as Series I and Series II. The design facilitates 3D structure formation and comprises three types of cells:

(a) Wheel mobile cell.

(b) Rotation joint cell.

(c) Bending joint cell.

The cells are fitted with castors at bottom for frictional less movement and are equipped with male and female connectors for docking. The wheel mobile cell shown in Figure 6 having mobile capabilities initiates docking with the

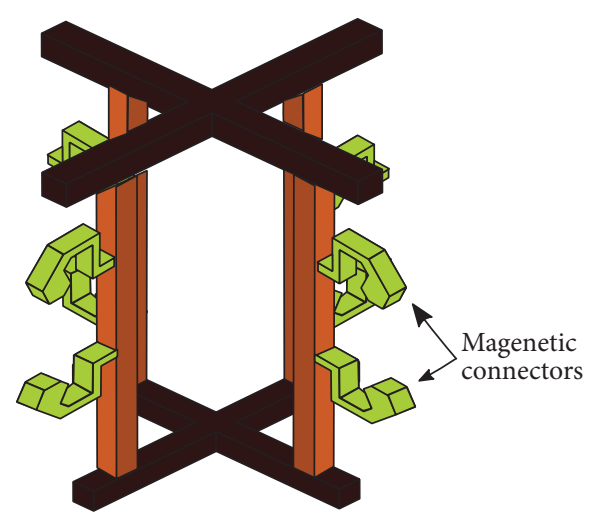

FIGURE 4: Model diagram of X-Bot MSRR.

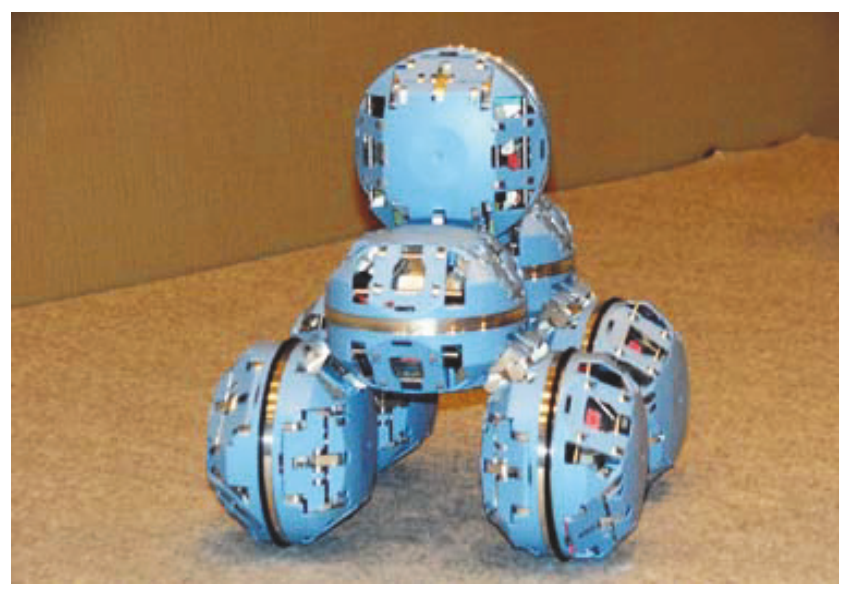

FIGURE 5: ATRON MSRR (http://modular.tek.sdu.dk/index.php? page $=$ robots).

TABLE 1: CEBOT, cell physical characteristics.

\begin{tabular}{lcc}
\hline & Series I & Series II \\
\hline Dimensions $(\mathrm{mm})$ & $190 * 90 * 50$ & $176 * 126 * 90$ \\
Weight $(\mathrm{Kg})$ & $1.2($ mobile cell $)$ & 2.7 (mobile cell) \\
& $1.2($ target cell $)$ & 1.0 (target cell) \\
Connectivity surface & Flat & Tapered \\
Coupler actuator & SMA & DC motor \\
\hline
\end{tabular}

necessary cell. The cells are equipped with SMA couplers for active latching of the male connector during docking and position sensors mounted on cells provide time to feedback on the docking process. The cells in series I require precise control and alignment for docking. The cells in series II are replaced with tapered female socket with worm gear for the active latch mechanism instead of SMA while maintaining the same docking process. The physical characteristics of cells in CEBOT are listed in Table 1.

Endo et al. developed ACM MSRR for mimicking snake alike chain structures in 2D. The ACM MSRR have three different versions: ACM [35], ACM-R2 [36], and ACMR3 [37]. Each unit in ACM is a wheeled square chassis robot without any actuator present for controlling individual 


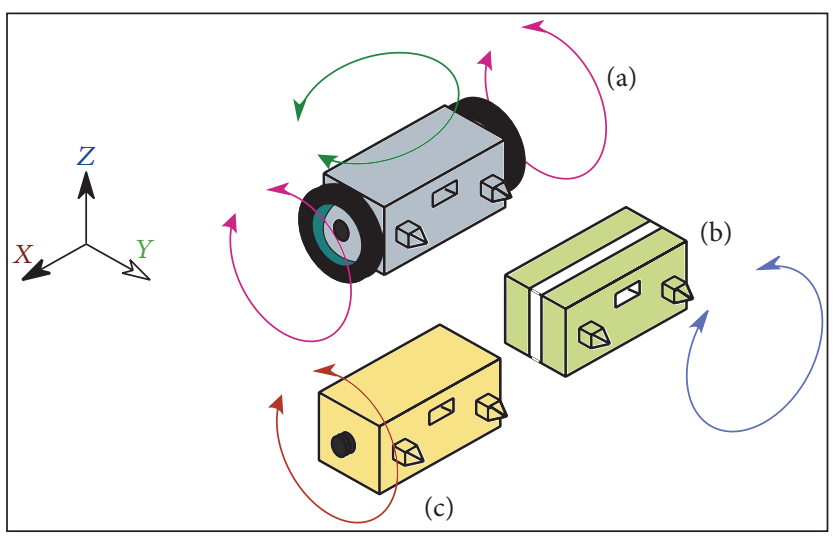

FIgURE 6: Structure of cells in CEBOT MSRR: (a) wheel mobile cell, (b) rotation joint cell, and (c) bending joint cell.

mobility. A servo motor is equipped behind every unit to rotate the robot at joint axis. The ACM MSRR is a combination of such individual homogeneous units assembled manually. The ACM-R2 is an improvement to ACM MSRR and has capabilities of forming 3D structures. The ACM-R2 MSRR is equipped with pitch and yaw motors in the joint unit between units for proving 2 DOF. The ACM-R3 is designed using custom frame body and wheels for providing robust support in formation of $3 \mathrm{D}$ structures and also facilitating manual assembly of robots with $\pm 90^{\circ}$ offsets with respect to each other.

Brown Jr. et al. prototyped a two-sided tracked vehicle called Millibot [38] capable of forming 2D structures for applications like movement in uneven terrains, stair climbing, and so forth. The Millibot MSRR is approximately an elliptical structure robot capable of self-docking using male and female connectors via latching mechanism actuated by SMA and is shown in Figure 7(a). The male connectors are installed in the front on a lifter capable of lifting objects vertically with the help of harmonic drives. Amoeba-I $[39,40]$ is another tracked MSRR with self-mobility proposed by Liu et al. for forming 3D structures. Each unit is a tracked elliptical structure capable of moving itself and is equipped with pitch joint on one side and yaw joint on the other. The robots when manually connected using physical links provide various DOF as shown in Figure 7(b). The amoebaI MSRR locomotion combinations are numerous depending on the orientation of link between the modules as well as actuation of corresponding joints. Li et al. developed improvised version of Millibot, JL 1 [41] and JL 2 [42], in terms of DOF by providing yaw and pitch control mechanism to each bot and also gear based docking mechanism at the cost of weight of robot. The major difference between JL-1 and JL2 is earlier employed latching mechanism for docking and later employed gripper for docking. The gripper on JL-2 can also be utilized as manipulator arm for holding objects in the environment. Lyder et al. published Thor [43] MSRR made up of modular blocks. The blocks are analytically developed motors, gears, right angle joints, gears, and wheels that can be utilized for forming various single robotic structures similar to Lego structures. The blocks can be assembled in various configurations due to symmetry in block designs and Thor is a robot build with a gripper using such blocks. Thor robot is equipped with wheels for mobility and gripper to dock with neighboring modules and so forth and hence making it a MSRR.

Yim designed Polypod [44] MSRR that falls under chain structure category and with capabilities of forming 3D structures. Polypod consists of two types of modules: segments and nodes. The nodes are rigid modules in cubical structure with single connector on each face providing six connectors from batteries. The segments are formed using 10-bar linkages providing two degree of freedom to the system and are capable of expanding or contracting in length as well as inclining towards left and right. The segments and nodes together facilitate formation of complex structures in 3D as shown in Figure 8.

The Polypod is actuated using small DC motor and position sensors are used for measuring angles of the linkages. The control architecture is implemented in three levels with highest level deciding the behavioral modes, the middle level executing the behavioral mode, and the lower level translating the commands to actuator joint space. The connection plates between the modules also facilitate the electrical connectivity for power and communications. In spite of absence of wheels the system is capable of movements like snake, caterpillar, rolling track turning, the moon walk dance, and so forth.

Castano et al. designed CONRO [45] MSSR to form structures like snakes or hexapods in $3 \mathrm{D}$. Each module in CONRO consists of three segments:

(a) Passive connector.

(b) Body.

(c) Active connector.

Two servo motors with rotation axis in orthogonal orientation are attached to the body as represented in Figure 9. The pitch motor is connected between the active connector and body. The yaw motor is connected to body and the passive connector. The docking mechanism and communication are handled using the feedback from IR transceivers present on the faces of active and passive connectors. The SMA equipped 


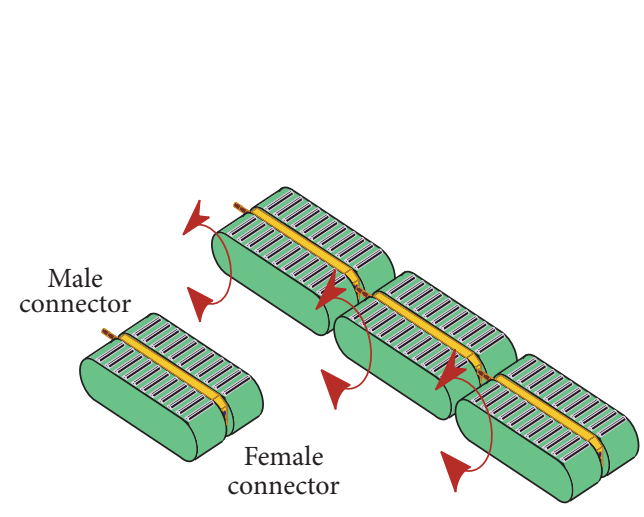

(a)

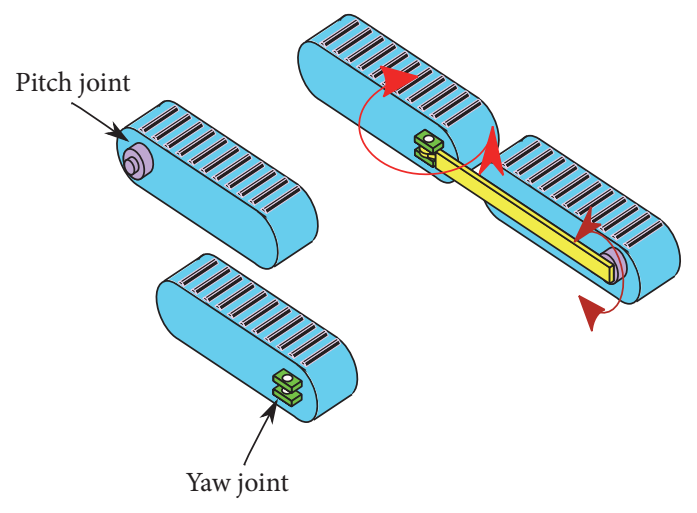

(b)

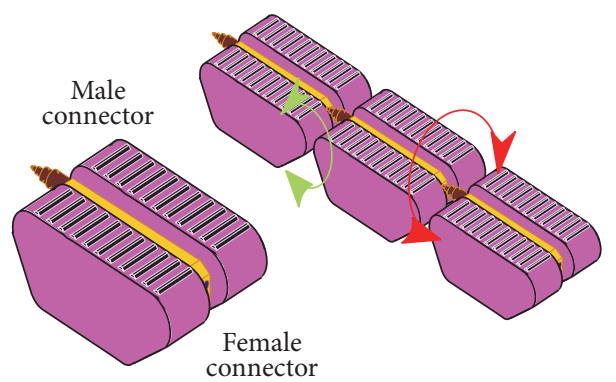

(c)

FIGURE 7: Chain structured mobile MSRR hardware models and structures: (a) Millibot, (b) Amoeba, and (c) JL-1.
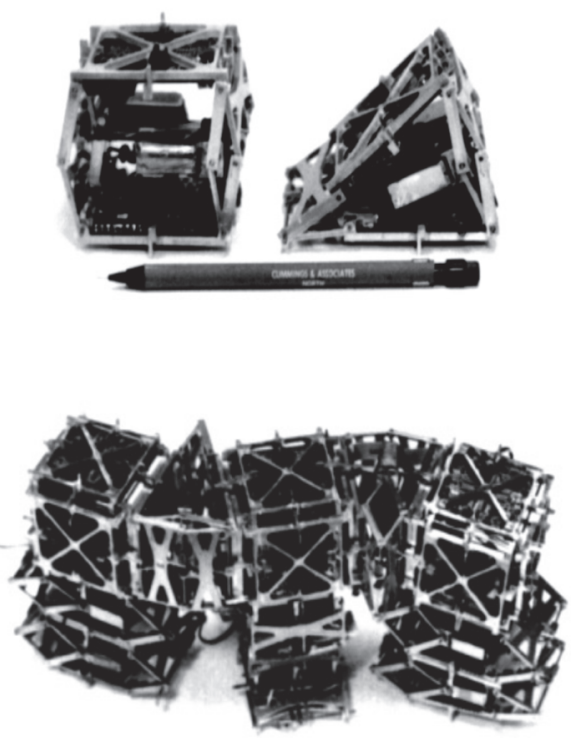

FIGURE 8: Polypod MSRR (source: http://robotics.stanford.edu/ users/mark/photos.html).

locking system present in passive connector latches the modules together after successful docking. A hormone based centralized and decentralized control for coordinate movements in modular robots was researched on CONRO robots in $[46,47]$. Further research on docking and alignment issues in CONRO robot modules are addressed in detail in [48].

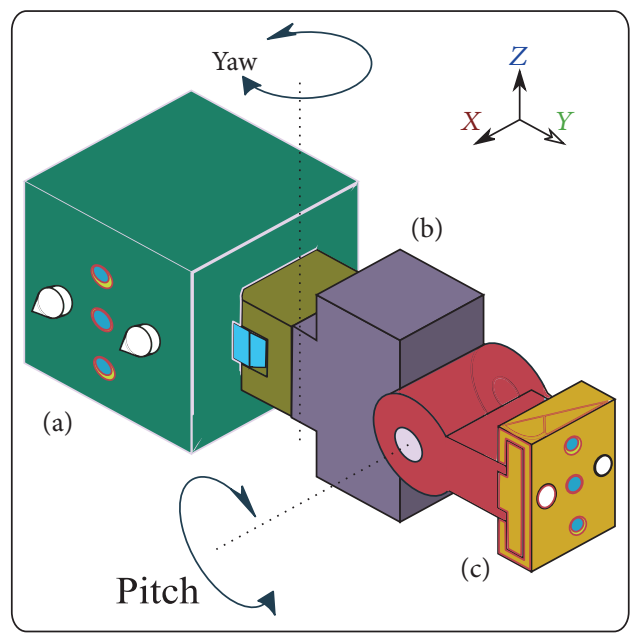

FIgURE 9: Model diagram of CONRO MSRR.

ModReD [49] MSRR proposed by Dasgupta et al. is similar to CONRO MSRR with modification in DOF. The ModRED robot consists of 3 cuboid blocks with 2 pitch motors: one at first block and the other at last block. A prismatic motor is placed along with pitch motor in last block for elongation of bond in horizontal plane between the center and last block. A roll motor is placed in center block for rotating front block with respect to center block. The first and last blocks are equipped with brackets as connectors with 
grooves and pins in structure of square along with a solenoid controlled mechanism for latching.

Polybot [50, 51] MSRR is a chain structure inspired robotic design capable of forming 3D structures. The Polypod is a cubic structure prototyped in three major versions: G1, G2, and G3. The G1 version of Polybot is a quick prototype with connection plates on front and back faces of $5 \mathrm{~cm}$ cube. The connection plates orientation with respect to each other can be changed with DC motor mounted outside the cube whose axis of rotation is normal to the side faces. The G1 prototype has no mechanism for latching and unlatching and hence docking is done manually. Since the connection plates are equipped with grooved pins and holes symmetrically, it is possible to dock two polybot G1 modules back to back even with $90^{\circ}$ offsets. The Polybot G2 is similar to G1 and additionally equipped with electromechanical latches and SMA controlled by software. The docking mechanism is guided by IR transceivers mounted on face plate and the robot is shown in Figure 10. The Polybot G3 are miniaturized modules with dimensions around $50 * 50 * 50 \mathrm{~mm}^{3}$. The externally visible DC motor in G1 and G2 version is made internally by changing the mechanism to $\mathrm{dc}$ pancake motor with harmonic gear along with active braking feature.

Transmote [52] module design is similar to Polybot with major difference in latching mechanism and number of connection surfaces. The front side face of transmote is equipped with a conical structure used for docking with female socket present at back of robot. The transmote facilitates twist and lock mechanism controlled by a servo motor for docking between robots. The transmote MSRR has connection provision on one side face along with front and back faces providing more stability to few $3 \mathrm{D}$ structures. The GZ-I MSRR robotic module proposed in [53] is similar to transmote with three connector faces and slightly different physical construction. The GZ-I modules are not equipped with docking sensors, actuators, and so forth and hence are assembled manually.

The YaMor [54] robot is a semicylindrical box structured robot capable of forming 2D chain structures. A triple beam in shape "ப" is connected to side faces of semicylindrical box at free ends of beams. Each robot module has one DOF and the system does not support autonomous docking. The velcros placed on beams, side faces, and back of the robots are used for docking with neighboring modules manually. The YaMor robot is a complete integrated solution with wireless communication capabilities and FPGA for reconfigurable computation purposes.

2.3. Hybrid Structured Systems. Mondada et al. developed a completely integrated autonomous robot called S-BOT [5562] capable of forming lattice structures in $2 \mathrm{D}$ and chain structures in $3 \mathrm{D}$ and hence a hybrid category robot. The robot is a cylindrical structured track robot designed for research in swarm robotics. The robots are capable of localization and navigation in uneven terrains. The robots employ gripper mechanism for docking with a ring covering the periphery of robot. Since the ring is present around the periphery, the docking can be done almost from every direction. The

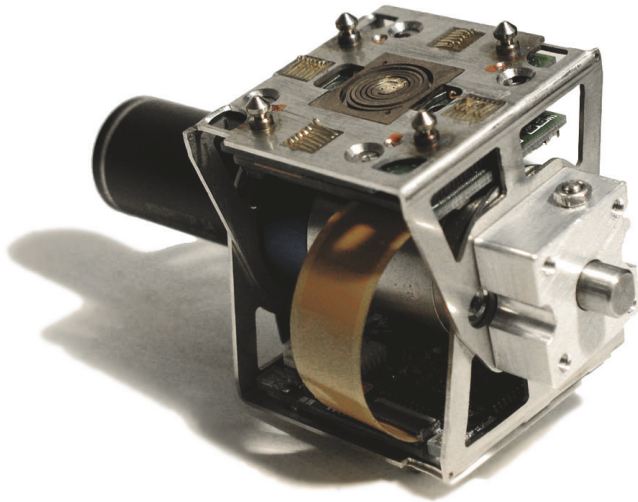

FIGURE 10: Polybot G2 MSRR (source: http://elek3ronik.blogspot.in/ 2007/05/chain-reconfiguration-robot.html).

optical sensors present in gripper modules form a closed control loop for providing feedback on docking process. The S-Bot employs the same features of modular robots such as modularity and reconfiguration.

$\mathrm{M}^{3}$ MSRR researched by Kutzer et al. is capable of forming 3D chain and lattice structures along with mobility features and is developed in two versions: $\mathrm{M}^{3}$ [63] and $\mathrm{M}^{3}$ express [64]. The modules are "L" shaped models with two wheels on parallel sides of long beam and one omnidirectional wheel on outside face of short beam parallel to surface and perpendicular to common rotational axis of two other wheels as shown in Figure 11. The wheels play dual role, enabling mobility and connection plates for docking. The $\mathrm{M}^{3}$ module is equipped with two hooks on wheels separated by $180^{\circ}$. The units are latched together when wheels of two modules come face to face with an offset of $180^{\circ}$ or $360^{\circ}$. The custom designed slip rings aid robots with docking as well as mobility using same wheels. In the $\mathrm{M}^{3}$ express module each wheel is equipped with two magnets at the ends of diameter, a yoke and four locking pins. The Yorks are connected to servo motors in a sliding mechanism for activating a slip disk with metallic screws. The disk is normally separated due to internal springs and the actuation of servo motor mounts the slip disk into wheels bringing metallic screws on to face of wheels at the ends of other diameters for docking.

Imobot $[65,66]$ is another mobile hybrid MSRR prototyped by Harry et al. The iMobot MSRR is cuboid structured formed from assembly of two semicylindrical modules as shown in Figure 12. The side faces of iMobot are equipped chamfered flat sheets capable of rotating continuously and hence providing mobile abilities to robot. The semicylindrical modules are capable of rotating $180^{\circ}$ along their axis independently. The four rotation mechanisms together aid iMobot to mimic movements such as crawling, rolling, and standing along with lattice and chain structures. The iMobot 


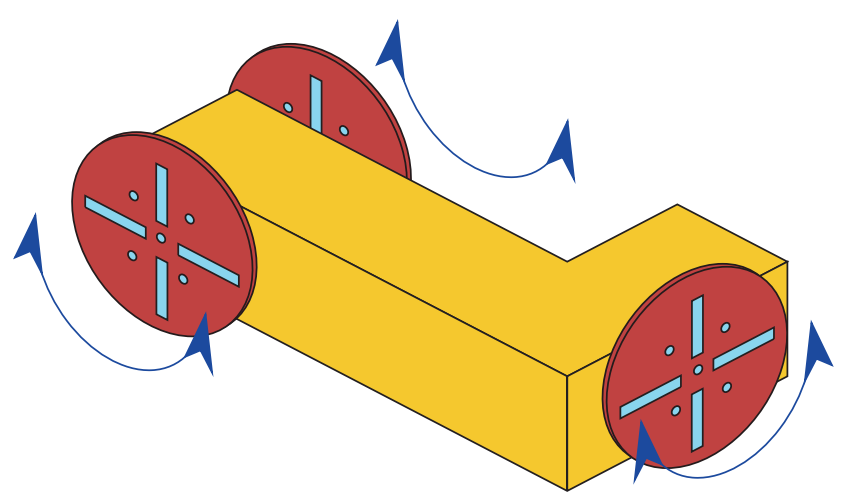

FIgURE 11: Model diagram of $\mathrm{M}^{3}$ MSRR.

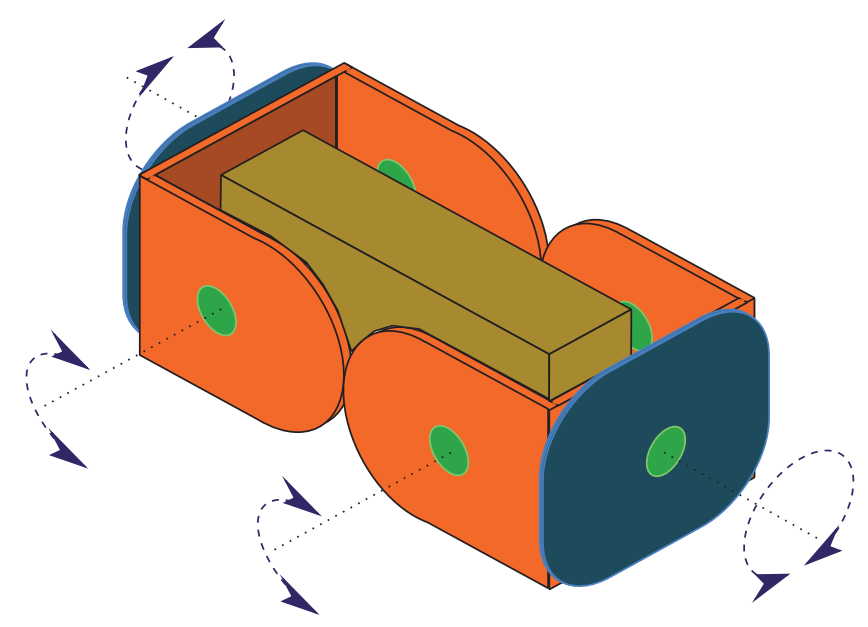

Figure 12: Model diagram of iMobot MSRR.

modules can assemble manually all the sides, hence forming various complex structures required for numerous real-time applications.

The SMORES [67] MSRR design is similar to iMobot consisting of a single semicylindrical cubic structure on which three of four side faces of cube are equipped with circular discs. Two circular discs on parallel faces play dual role in movement and docking and third for rolling neighboring modules after docking. Another internal motor provides pitch movement abilities to system by lifting third wheel. The locomotion is designed using orthogonally placed gears. Each face is equipped with four magnets with the same polarity magnets occupying alternate positions and hence at a time eight magnets participate in a docking when the connection plates face each other with an offset of $90^{\circ}$ or $270^{\circ}$. The docking keys selector present internally can extend through the center of all faces creating necessary gap for undocking.

Trimobot [68] is a fully integrated mobile category hexagonal MSRR capable of forming lattice structures in 2D and chain structures in 3D. The robot is equipped internally with three omnidirectional wheels on alternate sides of hexagonal structures for movement in 2D plane. The sides of trimobot are fixed with 5 passive connection faces and an active connection face on outside. A pitch joint is embedded with active connector face on one side of hexagonal structure to facilitate lifting of modules in vertical plane and hence forming chain structures in $3 \mathrm{D}$. The active connector face is also equipped with camera for docking purposes. The docking is enabled using four hooks present on active connector face and are controlled using rotation mechanism. The hooks are activated during docking when the passive and active connector faces of various modules face each other.

M-Tran is a hybrid configuration modular robot capable of forming 3D structures in both lattice and chain configurations and has three versions: M-Tran I $[69,70]$, M-Tran II [71, 72], and M-Tran III [73]. M-Tran robotics system consists of active and passive modules in the semicylindrical structures and a link permanently fixed in active unit as shown in Figure 13. The active and passive modules and links are equipped with four permanent magnets in a square structure on outside faces providing three connection surfaces on each module and two connection surfaces on link. The passive units can be coupled at the back of active units in two different angular orientations, $0^{\circ}$ and $360^{\circ}$ and $90^{\circ}$ and $270^{\circ}$, due to the alignment of magnets. The connection surfaces were also designed to aid electrical connectivity between the modules. The servo motors present in active unit enabled the rotation of link and the connection is established between units after a link present on active units enters the passive unit. The latching process is controlled by SMA coils by extending or retracting the magnets in passive units docked with magnets in link. The M-Tran II latches/unlatches link with passive part at $89 \%$ more efficiency in relation to M-Tran I with a tradeoff observed in time has improved torques and hardware used for sensing and control purposes. The M-Tran III is an improvised design in relation to previous versions. The latching/unlatching between link and passive part is replaced with hooks controlled by motor and hence providing more stable connection.

Superbot module proposed in $[74,75]$ is formed by permanently bonding two semicylindrical cells using link similar to iMobot MSRR. The cells are capable of rotating $180^{\circ}$ along their individual axis and also can roll with respect to bond binding them. The superbot MSRR has connectors on all faces making 6 connectors in total available for each superbot module. The rotating bond and two cells together provide 3 DOF to each superbot module: $180^{\circ}$ yaw, $180^{\circ}$ pitch, and $270^{\circ}$ roll. The superbot is capable of forming both lattice and chained structures and hence making it a hybrid category robot. The CKbot [76] MSRR design proposed by Yim et al. is similar to SMORES MSRR with reduction in self-mobility and rolling capabilities in individual units. The CKbot MSRR have autodocking/undocking features enabled by magnetic faces and also via screws if manual assembly is required and so forth. The CKbot MSRR is designed to test the self-healing capabilities of robotic system with the aid of vision after sudden events such as explosions.

Zykov et al. developed Molecubes [77], a cubic structure based hybrid category MSRR. The cube is assembly of two 


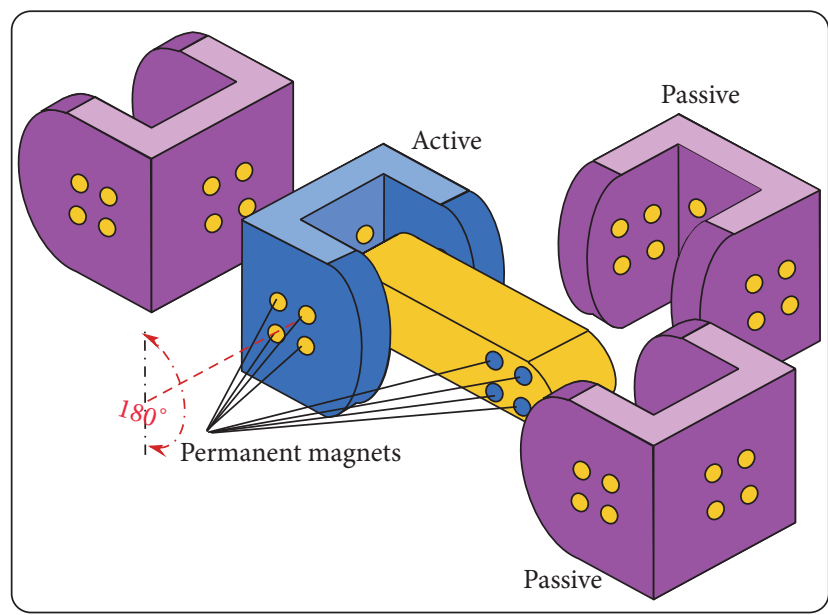

FIgURE 13: Model diagram of M-Tran MSRR.

parts made by splitting cubic structure of $10 \mathrm{~cm}$ along the plane perpendicular to a longest diagonal as shown in Figure 14. One-half of the cube can be rotated with respect to another in multiples of $120^{\circ}$ with the help of internal servo motor coupled with worm gear. The system is capable of forming both chained and lattice structures. The permanent pole magnets present around the center on faces facilitates coupling and the polarity of electromagnets at center can be utilized for severing or strengthening the bonds.

The UBot [78-80] MSRR system consists of cubic structured cells capable of rotating in discrete steps along longest diagonal similar to Molecubes. The internal faces are chamfered for facilitating rotation. The UBot robotic cells are categorized into active and passive modules with active modules providing four active connection interfaces and passive modules providing four passive connection interfaces. The active and passive modules have the same outer structures and rotation mechanisms. The hooks present on active connection interfaces enable firm docking with passive connectors. The active and passive modules are latched using hook and sliding mechanism guided by position sensors for forming lattice and chain structures in 3D making UBot a hybrid category robot.

Roombots [81, 82] MSRR is another hybrid architecture designed to from chained and lattice structure in 3D. Each roombot robot has two cells of spherical structure bonded together and each cell is a combination of two half-spheres mounted on each other along faces as shown in Figure 15. The locomotion is facilitated by three gear motors: one at the bond between cells and one is present in each cell for rotating other half spheres. Each roombot robot can be equipped with 10 active connections from neighboring modules to 1 active connection from a half sphere and 8 passive connections. The connection mechanism between various roombots is implemented with mechanical latches for holding neighboring modules at the holes present on surfaces.

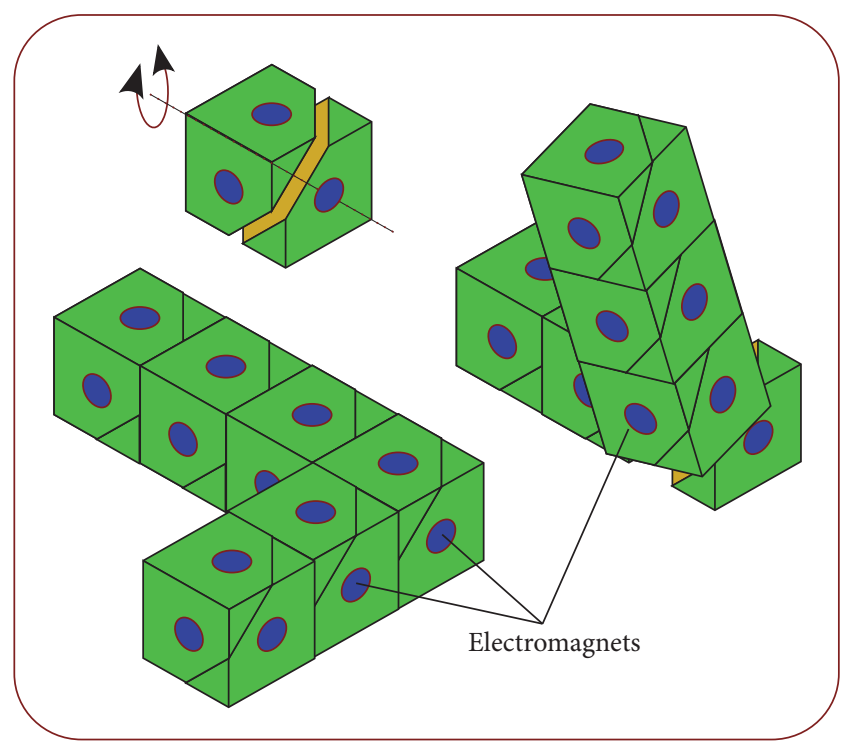

FIgURE 14: Model diagram and structures of Molecubes MSRR.

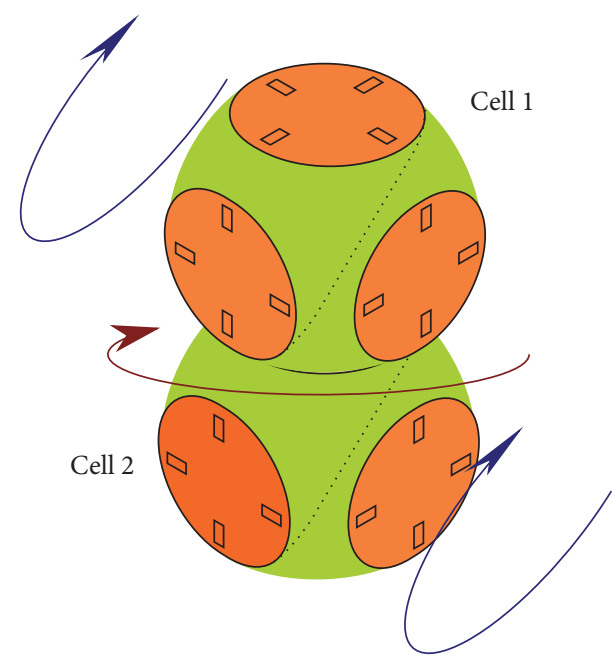

FIGURE 15: Model diagram of roombot MSRR.

Soldercubes $[83,84]$ developed by Neubert et al. is a hybrid category MSRR with shape of an individual Soldercube similar to a cell in dual-cell structure of roombots. The six genderless connector faces of each cell facilitate docking between modules and coordinate movement. The connector faces are custom made symmetrically designed PCB boards with soldering contacts. The contacts on connector faces can melt upon transmission of current at low temperatures, hence making a bond between modules for forming structures along with mechanical and electrical connections. The bond can be broken using the same mechanism of melting the contacts. The Soldercubes module has embedded mechanism for rotation of single connector face providing single DOF to the module but facilitating various DOF after docking with similar modules. 
2.4. Truss Structured Systems. Hamlin et al. prototyped a truss based MSRR-tetrobot [85, 86] for forming random structures using heterogeneous units: links and Joints. The links in tetrobot are cylindrical rods of fixed length and reconfiguration is supported only at the joints. A three-axis concentric multilink spherical joint capable of expansion and contraction in $3 \mathrm{D}$ is designed to hold three links together. The assembly between joints and links along with reconfiguration is performed by controlling joints using motors. Ramchurn et al. proposed the conceptual truss design MSRR-ORTHOBOT [87] with telescopic links having split toroids at two ends and with one toroid connected to link via revolute joint. The split-toroid joint aids in interconnectivity between modules providing 2 DOF rotation. The locomotion of coordinated system is simulated for structures such as hexapod.

Odin [88] MSRR consists of heterogeneous units: Cubic Closed Packed (CCP) joints and telescopic links along with capabilities to form structures in $3 \mathrm{D}$ as shown in Figure 16. The CCP has twelve female connector sockets each with internal female PCB connector. The telescopic links are extendable cylindrical structures with flexible connectors on both ends equipped with male PCB connectors. The modules are not capable of autonomous docking and are fitted manually. The joints act as power sharing and communication interfaces between the controllers present in links. The Morpho [89] truss system developed by $\mathrm{Yu}$ et al. consists of active links, passive links, and joints. The active links can expand and contract due to internal actuation of motors and the passive links expand and contract due to external forces. The links are joined together manually using cubic structured interfacing unit with a connector on each face. A surface membrane is covered over a 3D-skeleton structure formed using links and joints for realizing structures like conveyor belts with adapting topologies. Hjelle and Lipson developed Hinge MSRR [90] for reconfiguring truss structures. The design of truss system used as testbed is similar to odin MSRR. The joints have 18 female connectors and the struts are passive cylinders fastened by threaded inserts. Instead of providing locomotion in struts or joints, the Hinge robot maneuvers from one strut to another till it reaches destination and rotates the struts with help of servos by firmly holding them and hence reconfiguring the structure.

A concept of shape-shifting materials [91] was introduced by Amend and Lipson for programmable structures. The system consists of links and nodes like general truss systems. The links are beams of granular material instead of static metal structures. The nodes are connectors between the beams supporting transfer of granular materials from one beam to another. The nodes are capable of jamming the movement and hence modifying the stiffness of beams for changing structures. Galloway et al. developed a reconfigurable truss system called factory floor [92] to demonstrate the idea of autoassembly of truss structured systems. The CKBots equipped with a manipulator is used for assembly of custom structures by placing various elements together. The joints in factor floor MSRR are cubical structures with passive connectors on each face and the struts are hollow cuboid rods with grippers at both ends for docking. The pressing action

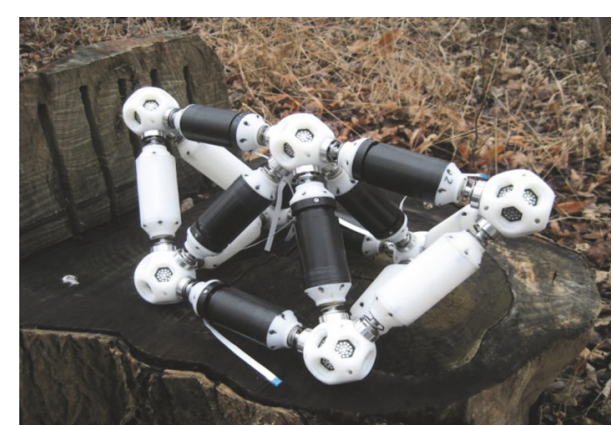

FIGURE 16: Odin MSRR (http://modular.tek.sdu.dk/index.php?page= robots).

performed by manipulator at the center of strut creates a couple force internally leading to opening of grippers.

\section{Free-Form Structured Systems}

Tokashiki et al. [93] prototyped a MSRR capable of forming free-form structures in 2D. The cylindrical structured MSRR (referred as Transform henceforth) is equipped with gear on top and bottom of the cylinder actuated by motors as shown in Figure 17. The robots are also equipped with 6 pole magnets around the periphery for providing bonding between the robots by attraction. The robots can move around when the gears of neighboring modules are locked with each other with magnets maintaining the structural integrity of the system. Goldstein et al. developed cylindrical structured MSRR named Claytronics [94-96] of diameter $44 \mathrm{~mm}$ for demonstrating various structures in 2D. The periphery of cylindrical structure is equipped with 24 spherical electromagnets in two rings present one below the other. The robots by themselves are immobile and require support of neighboring robots for forming structures as well as locomotion (on friction less surfaces). The modules have point contacts due to the shape of electromagnets and hence can implement various structures at much faster pace compared to other latched and rotating structures. Slime $[97,98]$ is another cylindrical design capable of forming free-form structure similar to Claytronics MSRR. The slime MSRR is equipped with 6 solenoids each controlling a $60^{\circ}$ section of $360^{\circ}$ periphery. Each cylinder section is equipped with a velcro to make contact with the neighboring robots. The spring action regulated by pneumatic air cylinders can extend and retract the cylinder sections for making and breaking the bond between robots. An extra solenoid placed downwards controls the position of a friction plate with respect to ground for increasing/decreasing friction during attachment/detachment process. The mini form factor MSRR, Catoms [99], is another cylindrical structure utilizing electrostatic forces for locomotion. The Catoms MSRR consists of a cylindrical wafer of $1 \mathrm{~mm}$ diameter and electrode strips placed vertically around the periphery of cylinder. The electrodes are sourced such that every alternate electrode holds charges of opposite polarities. The stability of structures is maintained by static fields and locomotion mechanism 


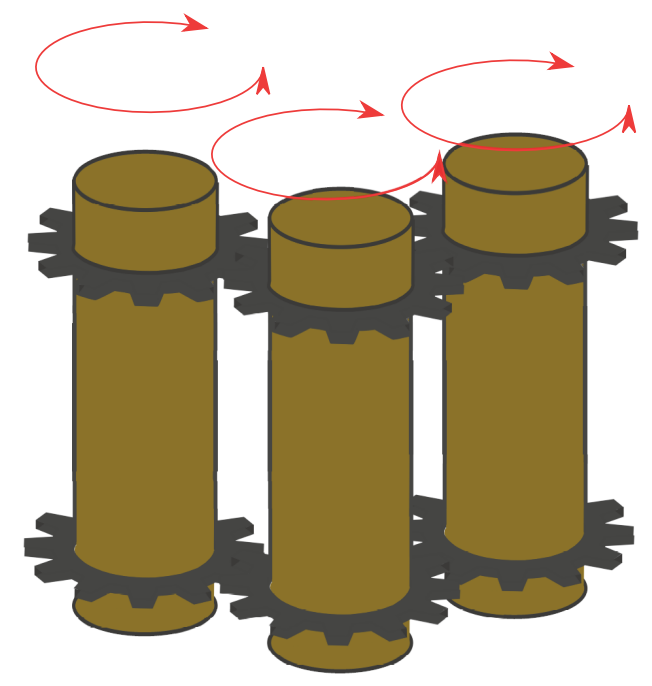

FIGURE 17: Architecture diagram of Transform MSRR.

is controlled by changing the polarities of electrodes on modules.

A micro form factor scratch drive MSRR, MEMS [100, 101], was developed by Donald et al. for forming free-form structures. The module consists of an arm and a scratch drive forming an " $\mathrm{L}$ " shaped structure whose structures are controlled by the voltages applied to module. The long beam acts as scratch drive for turning and the short beam in the structure is used for movements. The pulsating voltages applied to the system from bottom surface create various structures in arm and scratch drive with different frictional effects contributing to the movement. The authors explored various control algorithms and movement strategies for aligning the robots in a structure required using pulsating voltages.

The MSRR modules summarized so far are designed in various shapes such as squares and triangle for $2 \mathrm{D}$ scenarios and cube, cuboid, cylinder, and so forth for 3D scenarios so that the modules can have maximum contact surfaces for docking with neighbors while providing stability to coordinated structure as they adopt in the environment. The interfacing mechanism between individual MSRR modules for docking also plays vital role in restructuring the systems. Many researchers advocated the alignment issues raised during relocation of modules and stability of the interfaces for handling the increasing loads. Numerous sensor and actuator assemblies coupled with precise docking algorithms are attempted for autonomous docking of MSRR modules. The paradigm adopted for connection interfaces can be listed as male, female, active, and passive interfaces. The active connection interfaces are generally constructed using mechanical/electrical actuation assemblies for docking and the same are absent in passive connection interfaces. The passive connection interfaces still contribute to docking due to presence of passive materials like permanent magnets, sockets for screws, velcros, and so forth. The active and passive terminology is widely applied for genderless docking mechanisms and gender based docking designs differentiate between interfaces using male and female connection faces.

Table 2 provides a broad comparison of various MSRR robotic designs explained in the previous sections. The comparison is listed as per the categories mentioned in Figure 1. Since the shape generally defines the robustness of structures and the number of actuators along with type of actuator defines the parameters such as form factor and power consumption, the details of actuators and structures are also listed. The connection faces, structure, and number of connection faces on each MSRR module aid in identifying the probable structures possible when visualized in association with the shape of a robotic module. Since the connection faces are implemented using wide range of technologies, various jargons are adopted for categorizing them. The number of connection faces column in Table 2 lists details of a single robotic module in a MSRR design and is separated into two subcolumns: active and passive types for providing better visualization while interpreting locomotion capabilities. The entries in connection faces column for robotic module are listed as male (M), female (F), and dual role (DL, active and passive interfaces present on the same face). In the case of presence of heterogeneous modules in MSRR designs, the listed number is total of the active and passive interfaces present on heterogeneous units.

\section{Conclusion}

Research in MSRR has also extended to development of Robotic development environments, communication protocols (wired and wireless), middleware development [106, 107], human machine interface improvement, and so forth which are generally coupled with MSRR robotic modules providing a complete platform for rapid research in MSRR for development of algorithms, prototype validation, and so forth. The details on such platforms and protocols are not within scope of this paper and hence are not summarized.

In this paper a summary of various modular selfreconfigurable robotic structures is provided in terms of form factor, mobility, structural capabilities, and reconfiguration strategies. Research in MSRR as can be visualized from the summary is a deeply creative process employing technologies from mechanics and electronics and also requires deep understanding in merits/demerits of various sensor and actuation technologies. The research involves intensive prototyping and many MSRR models developed in past research with limited autonomous capabilities can be researched again due to availability of miniaturized sensor and actuator assemblies. This paper intends to provide preliminary study for prospective researchers by providing various innovations, strategies, and technologies employed in MSRR research.

\section{Conflicts of Interest}

The authors declare that they have no conflicts of interest. 


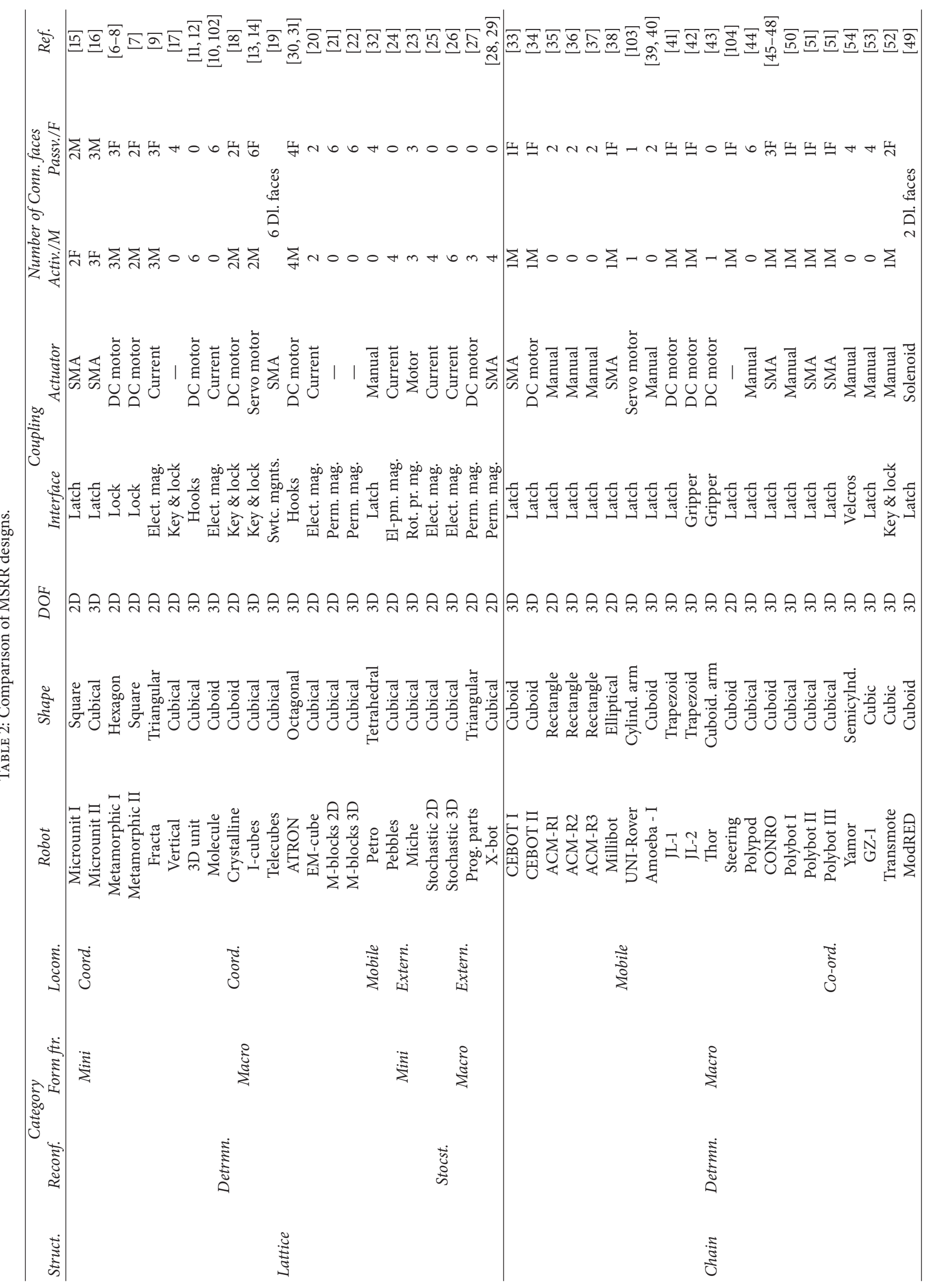




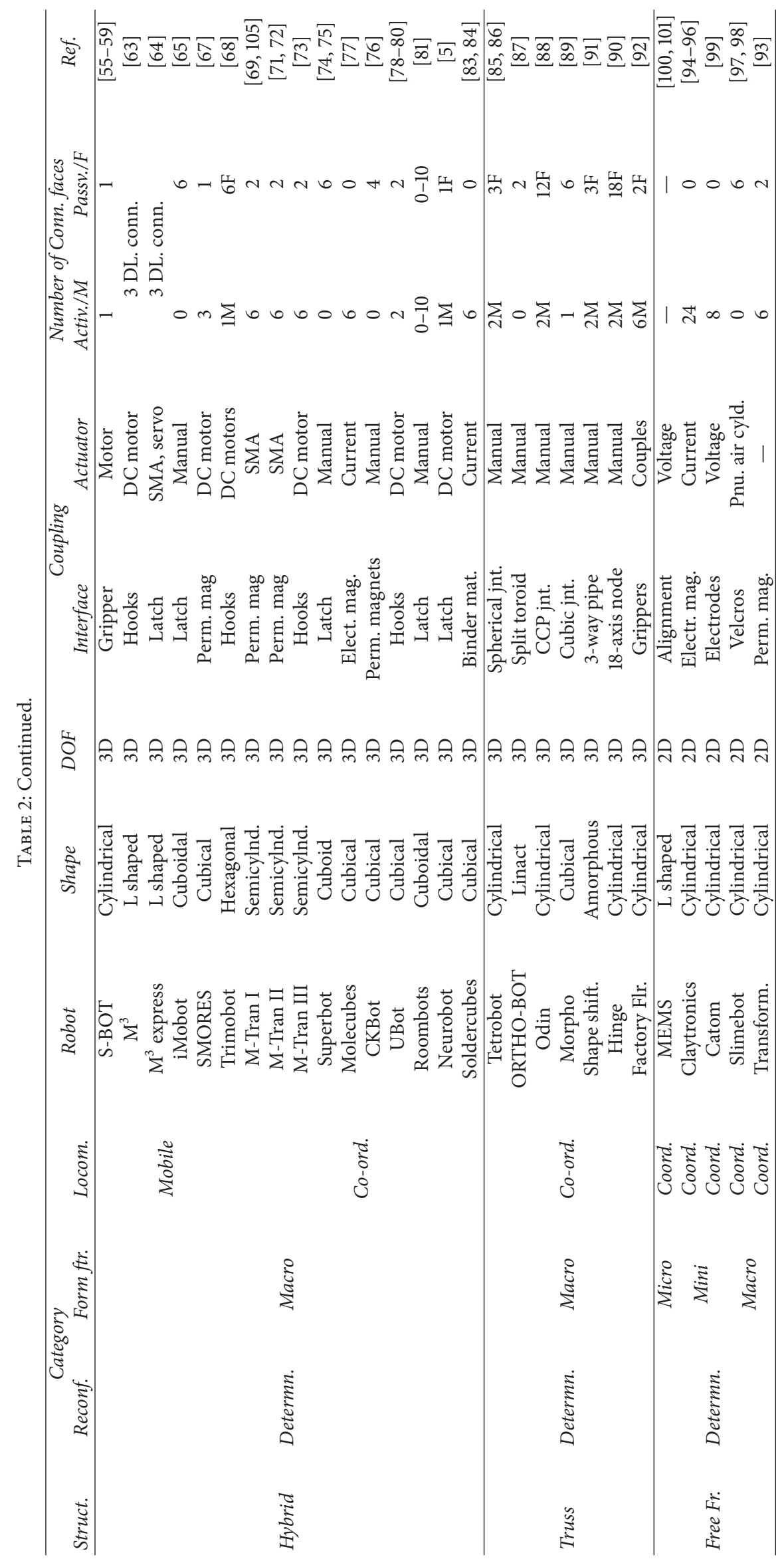




\section{References}

[1] J. Baca, P. Pagala, C. Rossi, and M. Ferre, "Modular robot systems towards the execution of cooperative tasks in large facilities," Robotics and Autonomous Systems, vol. 66, pp. 159174, 2015.

[2] M. Yim, W.-M. Shen, B. Salemi et al., "Modular selfreconfigurable robot systems [Grand challenges of robotics]," IEEE Robotics and Automation Magazine, vol. 14, no. 1, pp. 4352, 2007.

[3] M. Yim, P. White, M. Park, and J. Sastra, "Modular selfreconfigurable robots," in Encyclopedia of Complexity and Systems, pp. 19-33, Springer, New York, NY, USA, 2009.

[4] K. Gilpin and D. Rus, "Modular Robot Systems," IEEE Robotics \& Automation Magazine, vol. 17, no. 3, pp. 38-55, 2010.

[5] P. Moubarak and P. Ben-Tzvi, "Modular and reconfigurable mobile robotics," Robotics and Autonomous Systems, vol. 60, no. 12, pp. 1648-1663, 2012.

[6] G. S. Chirikjian, "Kinematics of a metamorphic robotic system," in Proceedings of the IEEE International Conference on Robotics and Automation, pp. 449-455, IEEE, San Diego, Calif, USA, May 1994.

[7] A. P. Chirikjian, D. Stein, C.-J. Chiang, and Grigory, "Design and Implementation of metamorphic robots," in Proceedings of the Design Engineering Technical Conference and Computers in Engineering Conference, pp. 1-10, ASME, Irvine, Calif, USA, 1996.

[8] A. Pamecha, I. Ebert-Uphoff, and G. S. Chirikjian, "Useful metrics for modular robot motion planning," IEEE Transactions on Robotics and Automation, vol. 13, no. 4, pp. 531-545, 1997.

[9] S. Murata, H. Kurokawa, and S. Kokaji, "Self-assembling machine," in Proceedings of the IEEE International Conference on Robotics and Automation, pp. 441-448, San Diego, Calif, USA, 1994.

[10] D. Rus, “Self-reconfiguring robots," IEEE Intelligent Systems, vol. 13, no. 4, pp. 2-4, 1998.

[11] H. Kurokawa, S. Murata, E. Yoshida, K. Tomita, and S. Kokaji, "A 3-D self-reconfigurable structure and experiments," in Proceedings of the IEEE/RSJ International Conference on Intelligent Robots and Systems. Innovations in Theory, Practice and Applications, vol. 2, pp. 860-865, Victoria, Canada, 1998.

[12] S. Murata, H. Kurokawa, E. Yoshida, K. Tomita, and S. Kokaji, "A 3-D self-reconfigurable structure," in Proceedings of the IEEE International Conference on Robotics and Automation, vol. 1, pp. 432-439, IEEE, Leuven, Belgium, May 1998.

[13] C. Ünsal, H. Kiliççöte, and P. K. Khosla, "Modular selfreconfigurable bipartite robotic system: implementation and motion planning," Autonomous Robots, vol. 10, no. 1, pp. 23-40, 2001.

[14] C. Unsal and P. Khosla, "Mechatronic design of a modular selfreconfiguring robotic system," in Proceedings of the Millennium Conference on IEEE International Conference on Robotics and Automation (ICRA '00), vol. 2, pp. 1742-1747, IEEE, April 2000, Symposia Proceedings Cat. No. 00CH37065.

[15] E. Yoshida, S. Murata, S. Kokaji, K. Tomita, and H. Kurokawa, "Micro self-reconfigurable robotic system using shape memory alloy," in Proceedings of the Symposium on Distributed Autonomous Robotic Systems (DARS '00), vol. 4, pp. 145-154, Springer, Tokyo, Japan, 2000.

[16] E. Yoshida, S. Murata, S. Kokaji, A. Kamimura, K. Tomita, and H. Kurokawa, "Get back in shape! [SMA self-reconfigurable microrobots]," IEEE Robotics \& Automation Magazine, vol. 9, no. 4, pp. 54-60, 2002.

[17] K. Hosokawa, T. Tsujimori, T. Fujii et al., "Self-organizing collective robots with morphogenesis in a vertical plane," in Proceedings of the IEEE International Conference on Robotics and Automation, pp. 2858-2863, May 1998.

[18] D. Rus and M. Vona, "A physical implementation of the selfreconfiguring crystalline robot," in Proceedings of the IEEE International Conference on Robotics and Automation (ICRA '00), vol. 2, pp. 1726-1733, San Francisco, Calif, USA, April 2000.

[19] J. Suh, S. Homans, and M. Yim, "Telecubes: mechanical design of a module for self-reconfigurable robotics," in Proceedings of the IEEE International Conference on Robotics and Automation, pp. 4095-4101, IEEE, Washington, DC, USA, May 2002.

[20] B. K. An, "EM-Cube: cube-shaped, self-reconfigurable robots sliding on structure surfaces," in Proceedings of the IEEE International Conference on Robotics and Automation (ICRA '08), pp. 3149-3155, Pasadena, Calif, USA, May 2008.

[21] J. W. Romanishin, K. Gilpin, and D. Rus, "M-blocks: momentum-driven, magnetic modular robots," in Proceedings of the 26th IEEE/RSJ International Conference on Intelligent Robots and Systems (IROS '13), pp. 4288-4295, IEEE, Tokyo, Japan, November 2013.

[22] J. W. Romanishin, K. Gilpin, S. Claici, and D. Rus, “3D MBlocks: self-reconfiguring robots capable of locomotion via pivoting in three dimensions," in Proceedings of the IEEE International Conference on Robotics and Automation (ICRA '15), pp. 1925-1932, Seattle, Wash, USA, May 2015.

[23] K. Gilpin, K. Kotay, and D. Rus, "Miche: modular shape formation by self-dissasembly," in Proceedings of the IEEE International Conference on Robotics and Automation (ICRA '07), pp. 2241-2247, IEEE, Roma, Italy, April 2007.

[24] K. Gilpin, A. Knaian, and D. Rus, "Robot pebbles: one centimeter modules for programmable matter through selfdisassembly," in Proceedings of the IEEE International Conference on Robotics and Automation (ICRA '10), pp. 2485-2492, May 2010.

[25] P. White, K. Kopanski, and H. Lipson, "Stochastic selfreconfigurable cellular robotics," in Proceedings of the IEEE International Conference on Robotics and Automation (ICRA '04), vol. 3, pp. 2888-2893, New Orleans, La, USA, April 2004.

[26] P. White, V. Zykov, J. Bongard, and H. Lipson, "Three dimensional stochastic reconfiguration of modular robots," in Proceedings of the International Conference on Robotics: Science and Systems (RSS '05), pp. 161-168, June 2005.

[27] J. Bishop, S. Burden, E. Klavins et al., "Programmable parts: a demonstration of the grammatical approach to selforganization," in Proceedings of the IEEE/RSJ International Conference on Intelligent Robots and Systems, pp. 3684-3691, IEEE, Alberta, Canada, August 2005.

[28] P. J. White and M. Yim, "Scalable modular self-reconfigurable robots using external actuation," in Proceedings of the IEEE/RSJ International Conference on Intelligent Robots and Systems (IROS '07), pp. 2773-2778, November 2007.

[29] P. J. White and M. Yim, "Reliable external actuation for extending reachable robotic modular self-reconfiguration," in Proceedings of the 11th International Symposium on Experimental Robotics, pp. 13-23, Athens, Greece, 2009.

[30] M. W. Jørgensen, E. H. Østergaard, and H. H. Lund, "Modular ATRON: modules for a self-reconfigurable robot," in Proceedings of the IEEE/RSJ International Conference on Intelligent 
Robots and Systems (IROS '04), pp. 2068-2073, IEEE, Sendai, Japan, October 2004.

[31] U. P. Schultz, M. Bordignon, and K. Stoy, "Robust and reversible self-reconfiguration," in Proceedings of the IEEE/RSJ International Conference on Intelligent Robots and Systems (IROS '09), pp. 5287-5294, October 2009.

[32] B. Salem, "PetRo: development of a modular pet robot," in Proceedings of the 23rd IEEE International Symposium on Robot and Human Interactive Communication, pp. 483-488, IEEE, August 2014.

[33] T. Fukuda and S. Nakagawa, "Dynamically reconfigurable robotic system," in Proceedings of the IEEE International Conference on Robotics and Automation, pp. 1581-1586, IEEE Computer Society Press, Philadelphia, Pa, USA, 1988.

[34] T. Fukuda and S. Nakagawa, "Method of autonomous approach, docking and detaching between cells for dynamically reconfigurable robotic system CEBOT," JSME International Journal. Series 3: Vibration, Control Engineering, Engineering for Industry, vol. 33, no. 2, pp. 263-268, 1990, http://ci.nii.ac.jp/ naid/130003964367/en/.

[35] G. Endo, K. Togawa, and S. Hirose, "Study on self-contained and terrain adaptive active cord mechanism," in Proceedings of the IEEE/RSJ International Conference on Intelligent Robots and Systems. Human and Environment Friendly Robots with High Intelligence and Emotional Quotients (Cat. No.99CH36289), vol. 3, no. 1, pp. 1399-1405, Kyongju, South Korea, 1999.

[36] K. Togawa, M. Mori, and S. Hirose, "Study on threedimensional active cord mechanism: development of ACMR2," in Proceedings of the IEEE/RSJ International Conference on Intelligent Robots and Systems (IROS '00), vol. 3, pp. 2242-2247, November 2000.

[37] M. Mori and S. Hirose, "Development of active cord mechanism ACM-R3 with agile 3D mobility," in Proceedings of the IEEE/RSJ International Conference on Intelligent Robots and Systems, vol. 3, pp. 1552-1557, IEEE, Maui, Hawaii, USA, 2001.

[38] H. B. Brown Jr., J. M. Vande Weghe, C. A. Bererton, and P. K. Khosla, "Millibot trains for enhanced mobility," IEEE/ASME Transactions on Mechatronics, vol. 7, no. 4, pp. 452-461, 2002.

[39] L. Jinguo, M. Shugen, L. Zhenli, W. Yuechao, L. Bin, and W. Jing, "Design and experiment of a novel link-type shape shifting modular robot series," in Proceedings of the IEEE International Conference on Robotics and Biomimetics (ROBIO '05), pp. 318323, July 2005.

[40] B. Li, S. Ma, J. Liu, M. Wang, T. Liu, and Y. Wang, "AMOEBAI: a shape-shifting modular robot for urban search and rescue," Advanced Robotics, vol. 23, no. 9, pp. 1057-1083, 2009.

[41] D. Li, H. Fu, and W. Wang, "Ultrasonic based autonomous docking on plane for mobile robot," in Proceedings of the IEEE International Conference on Automation and Logistics (ICAL '08), pp. 1396-1401, IEEE, Qingdao, China, September 2008.

[42] W. Wang, W. Yu, and H. Zhang, "JL-2: a mobile multi-robot system with docking and manipulating capabilities," International Journal of Advanced Robotic Systems, vol. 7, no. 1, pp. 9-18, 2010.

[43] A. Lyder, R. Franco, M. Garcia, and K. Stoy, "Genderless connection mechanism for modular robots introducing torque transmission between modules," in Proceedings of the ICRA 2010 Workshop on Modular Robots: The State of the Art, pp. 77-82, 2010.

[44] M. Yim, "New locomotion gaits," in Proceedings of the IEEE International Conference on Robotics and Automation, pp. 25082514, IEEE Computer Society Press, San Diego, Calif, USA, 1994.
[45] A. Castano, R. Chokkalingam, and P. Will, "Autonomous and self-sufficient CONRO Modules for Recon gurable robots," in Distributed Autonomous Robotic Systems 4, chapter 5, pp. 155164, Springer, Tokyo, Japan, 2000.

[46] W.-M. Shen, B. Salemi, and P. Will, "Hormone-inspired adaptive communication and distributed control for CONRO selfreconfigurable robots," IEEE Transactions on Robotics and Automation, vol. 18, no. 5, pp. 700-712, 2002.

[47] W.-M. Shen, Y. Lu, and P. Will, "Hormone-based control for self-reconfigurable robots," in Proceedings of the 4th International Conference on Autonomous Agents (AGENTS '00), vol. 00, pp. 1-8, 2000, http://portal.acm.org/citation.cfm?doid= 336595.336602.

[48] M. Rubenstein, K. Payne, P. Will, and W.-M. Shen, "Docking among independent and autonomous CONRO selfreconfigurable robots," in Proceedings of the IEEE International Conference on Robotics and Automation (ICRA '04), vol. 3, pp. 2877-2882, May 2004.

[49] P. Dasgupta, J. Baca, A. Dutta, S. M. G. Hossain, and C. Nelson, "Mechanical design and computational aspects for locomotion and reconfiguration of the ModRED Modular Robot," in Proceedings of the 12th International Conference on Autonomous Agents and Multiagent Systems (AAMAS '13), pp. 1359-1360, ACM, Saint Paul, Minn, USA, May 2013.

[50] M. Yim, D. Duff, and K. Roufas, "PolyBot: a modular reconfigurable robot," in Proceedings of the IEEE International Conference on Robotics and Automation (ICRA '00), vol. 1, pp. 514-520, San Francisco, Calif, USA, 2000.

[51] M. Yim, Y. Zhang, K. Roufas, D. Duff, and C. Eldershaw, "Connecting and disconnecting for chain self-reconfiguration with polybot," IEEE/ASME Transactions on Mechatronics, vol. 7, no. 4, pp. 442-451, 2002.

[52] G. Qiao, G. Song, J. Zhang, H. Sun, W. Wang, and A. Song, "Design of transmote: a modular self-reconfigurable robot with versatile transformation capabilities," in Proceedings of the IEEE International Conference on Robotics and Biomimetics (ROBIO '12), pp. 1331-1336, December 2012.

[53] Y. Li, H. Zhang, and S. Chen, "A four-legged robot based on GZ-I modules," in Proceedings of the IEEE International Conference on Robotics and Biomimetics (ROBIO '08), pp. 921926, Bangkok, Thailand, February 2009.

[54] R. Moeckel, C. Jaquier, K. Drapel, E. Dittrich, A. Upegui, and A. J. Ijspeert, "Exploring adaptive locomotion with YaMoR, a novel autonomous modular robot with Bluetooth interface," Industrial Robot, vol. 33, no. 4, pp. 285-290, 2006.

[55] F. Mondada, G. C. Pettinaro, A. Guignard et al., "Swarm-bot: a new distributed robotic concept," Autonomous Robots, vol. 17, no. 2-3, pp. 193-221, 2004.

[56] M. Dorigo, "SWARM-BOT: an experiment in swarm robotics," in Proceedings of the IEEE Swarm Intelligence Symposium (SIS '05), pp. 192-200, IEEE, Pasadena, Calif, USA, June 2005.

[57] E. Tuci, R. Gross, V. Trianni, F. Mondada, M. Bonani, and M. Dorigo, "Cooperation through self-assembly in multi-robot systems," ACM Transactions on Autonomous and Adaptive Systems, vol. 1, no. 2, pp. 115-150, 2006.

[58] R. Gro, M. Bonani, F. Mondada, and M. Dorigo, "Autonomous self-assembly in swarm-bots," IEEE Transactions on Robotics, vol. 22, no. 6, pp. 1115-1130, 2006.

[59] R. Groß, E. Tuci, M. Dorigo, M. Bonani, and F. Mondada, "Object transport by modular robots that self-assemble," in Proceedings of the IEEE International Conference on Robotics and Automation (ICRA '06), pp. 2558-2564, May 2006. 
[60] V. Trianni, S. Nolfi, and M. Dorigo, "Cooperative hole avoidance in a swarm-bot," Robotics and Autonomous Systems, vol. 54, no. 2, pp. 97-103, 2006.

[61] R. O'Grady, R. Groß, A. L. Christensen, F. Mondada, M. Bonani, and M. Dorigo, "Performance benefits of self-assembly in a swarm-bot," in Proceedings of the IEEE/RSJ International Conference on Intelligent Robots and Systems (IROS '07), pp. 2381-2387, November 2007.

[62] G. Fu, A. Menciassi, and P. Dario, "Development of a genderless and fail-safe connection system for autonomous modular robots," in Proceedings of the IEEE International Conference on Robotics and Biomimetics (ROBIO '11), pp. 877-882, IEEE, Karon Beach, Thailand, December 2011.

[63] M. D. M. Kutzer, M. S. Moses, C. Y. Brown, D. H. Scheidt, G. S. Chirikjian, and M. Armand, "Design of a new independentlymobile reconfigurable modular robot," in Proceedings of the IEEE International Conference on Robotics and Automation (ICRA '10), pp. 2758-2764, May 2010.

[64] K. C. Wolfe, M. S. Moses, M. D. M. Kutzer, and G. S. Chirikjian, " $\mathrm{M}^{3}$ Express: a low-cost independently-mobile reconfigurable modular robot," in Proceedings of the IEEE International Conference on Robotics and Automation, pp. 2704-2710, IEEE, St. Paul, Minn, USA, May 2012.

[65] G. G. Ryland and H. H. Cheng, "Design of iMobot, an intelligent reconfigurable mobile robot with novel locomotion," in Proceedings of the IEEE International Conference on Robotics and Automation (ICRA '10), pp. 60-65, May 2010.

[66] D. Ko and H. H. Cheng, "Programming reconfigurable modular robots," in Proceedings of the 8th IEEE/ASME International Conference on Mechatronic and Embedded Systems and Applications (MESA '12), pp. 160-165, July 2012.

[67] J. Davey, N. Kwok, and M. Yim, "Emulating self-reconfigurable robots-design of the SMORES system," in Proceedings of the 25th IEEE/RSJ International Conference on Robotics and Intelligent Systems (IROS '12), pp. 4464-4469, October 2012.

[68] Y. Zhang, G. Song, S. Liu, G. Qiao, J. Zhang, and H. Sun, "A modular self-reconfigurable robot with enhanced locomotion performances: design, modeling, simulations, and experiments," Journal of Intelligent and Robotic Systems: Theory and Applications, vol. 81, no. 3-4, pp. 377-393, 2016.

[69] S. Murata, E. Yoshida, A. Kamimura, H. Kurokawa, K. Tomita, and S. Kokaji, "M-TRAN: self-reconfigurable modular robotic system," IEEE/ASME Transactions on Mechatronics, vol. 7, no. 4, pp. 431-441, 2002.

[70] E. Yoshida, S. Murata, A. Kamimura, K. Tomita, H. Kurokawa, and S. Kokaji, "A motion planning method for a selfreconfigurable modular robot," in Proceedings of the RSJ/IEEE International Conference on Intelligent Robots and Systems, vol. 1, pp. 590-597, Maui, Hawaii, USA, 2000.

[71] H. Kurokawa, A. Kamimura, E. Yoshida, K. Tomita, S. Kokaji, and S. Murata, "M-TRAN II: metamorphosis from a four-legged walker to a caterpillar," in Proceedings of the IEEE/RSJ International Conference on Intelligent Robots and Systems (IROS '03), vol. 3, pp. 2454-2459, Las Vegas, Nev, USA, October 2003.

[72] A. Kamimura, H. Kurokawa, E. Yoshida, K. Tomita, S. Kokaji, and S. Murata, "Distributed adaptive locomotion by a modular robotic system, M-TRAN II," in Proceedings of the IEEE/RSJ International Conference on Intelligent Robots and Systems (IROS '04), vol. 3, pp. 2370-2377, IEEE, Septemper-October 2004, IEEE Cat. No. 04CH37566.

[73] H. Kurokawa, K. Tomita, A. Kamimura, S. Kokaji, T. Hasuo, and S. Murata, "Distributed self-reconfiguration of M-TRAN
III modular robotic system," International Journal of Robotics Research, vol. 27, no. 3-4, pp. 373-386, 2008.

[74] B. Salemi, M. Moll, and W.-M. Shen, "SUPERBOT: a deployable, multi-functional, and modular self-reconfigurable robotic system," in Proceedings of the IEEE/RSJ International Conference on Intelligent Robots and Systems (IROS '06), pp. 3636-3641, October 2006.

[75] W.-M. Shen, M. Krivokon, H. Chiu, J. Everist, M. Rubenstein, and J. Venkatesh, "Multimode locomotion via SuperBot robots," in Proceedings of the IEEE International Conference on Robotics and Automation (ICRA '06), pp. 2552-2557, Orlando, Fla, USA, May 2006.

[76] M. Yim, B. Shirmohammadi, J. Sastra, M. Park, M. Dugan, and C. J. Taylor, "Towards robotic self-reassembly after explosion," in Proceedings of the IEEE/RSJ International Conference on Intelligent Robots and Systems (IROS '07), pp. 2767-2772, IEEE, San Diego, Calif, USA, November 2007.

[77] V. Zykov, E. Mytilinaios, M. Desnoyer, and H. Lipson, "Evolved and designed self-reproducing modular robotics," IEEE Transactions on Robotics, vol. 23, no. 2, pp. 308-319, 2007.

[78] S. T. S. Tang, Y. Z. Y. Zhu, J. Z. J. Zhao, and X. C. X. Cui, “The UBot modules for self-reconfigurable robot," in Proceedings of the ASME/IFToMM International Conference on Reconfigurable Mechanisms and Robots (ReMAR '09), pp. 529-535, June 2009.

[79] Y. Zhu, J. Zhao, X. Cui et al., "Design and implementation of UBot: a modular self-reconfigurable robot," in Proceedings of the 10th IEEE International Conference on Mechatronics and Automation (ICMA '13), pp. 1217-1222, IEEE, Takamatsu, Japan, August 2013.

[80] Y. Zhu, H. Jin, X. Zhang, J. Yin, P. Liu, and J. Zhao, "A multisensory autonomous docking approach for a self-reconfigurable robot without mechanical guidance," International Journal of Advanced Robotic Systems, vol. 11, 2014.

[81] A. Sproewitz, A. Billard, P. Dillenbourg, and A. J. Ijspeert, "Roombots-mechanical design of self-reconfiguring modular robots for adaptive furniture," in Proceedings of the IEEE International Conference on Robotics and Automation (ICRA '09), pp. 4259-4264, IEEE, Kobe, Japan, May 2009.

[82] A. Sproewitz, P. Laprade, S. Bonardi et al., "Roombots-towards decentralized reconfiguration with self-reconfiguring modular robotic metamodules," in Proceedings of the 23rd IEEE/RSJ International Conference on Intelligent Robots and Systems (IROS '10), pp. 1126-1132, October 2010.

[83] J. Neubert, A. Rost, and H. Lipson, "Self-soldering connectors for modular robots," IEEE Transactions on Robotics, pp. 1-14, 2014.

[84] J. Neubert and H. Lipson, "Soldercubes: a self-soldering selfreconfiguring modular robot system," Autonomous Robots, vol. 40, no. 1, pp. 139-158, 2016.

[85] G. J. Hamlin and A. C. Sanderson, "Tetrobot: a modular system for hyper-redundant parallel robotics," in Proceedings of the IEEE International Conference on Robotics and Automation 1995, May 1995.

[86] G. J. Hamlin and A. C. Sanderson, “TETROBOT: a modular approach to parallel robotics," IEEE Robotics and Automation Magazine, vol. 4, no. 1, p. 42, 1997.

[87] V. Ramchurn, R. C. Richardson, and P. Nutter, "ORTHO-BOT: a modular reconfigurable space robot concept," in Climbing and Walking Robots, pp. 659-666, Springer, Berlin, Germany, 2006.

[88] A. Lyder, R. F. M. Garcia, and K. Stoy, "Mechanical design of Odin, an extendable heterogeneous deformable modular 
robot," in Proceedings of the IEEE/RSJ International Conference on Intelligent Robots and Systems (IROS '08), pp. 883-888, IEEE, Nice, France, September 2008.

[89] C.-H. Yu, K. Haller, D. Ingber, and R. Nagpal, "Morpho: a selfdeformable modular robot inspired by cellular structure," in Proceedings of the IEEE/RSJ International Conference on Intelligent Robots and Systems (IROS '08), pp. 3571-3578, September 2008.

[90] D. Hjelle and H. Lipson, "A robotically reconfigurable truss," in Proceedings of the ASME/IFToMM International Conference on Reconfigurable Mechanisms and Robots (ReMAR '09), pp. 73-78, June 2009.

[91] J. R. Amend and H. Lipson, "Shape-shifting materials for programmable structures," in Proceedings of the in International Conference on Ubiquitous Computing: Workshop on Architectural Robotics, September-October 2009.

[92] K. C. Galloway, R. Jois, and M. Yim, "Factory floor: a robotically reconfigurable construction platform," in Proceedings of the IEEE International Conference on Robotics and Automation (ICRA '10), pp. 2467-2472, IEEE, May 2010.

[93] H. Tokashiki, H. Amagai, S. Endo, K. Yamada, and J. Kelly, "Development of a transformable mobile robot composed of homogeneous gear-type units," in Proceedings of the IEEE/RSJ International Conference on Intelligent Robots and Systems (IROS '03), vol. 2, pp. 1602-1607, October 2003.

[94] S. Goldstein and T. Mowry, "Claytronics: an instance of programmable matter," in Proceedings of the Wild and Crazy Ideas Session of ASPLOS, vol. 17, Boston, Mass, USA, October 2004.

[95] S. C. Goldstein, J. D. Campbell, and T. C. Mowry, "Programmable matter," Computer, vol. 38, no. 6, pp. 99-101, 2005.

[96] J. Campbell, P. Pillai, and S. C. Goldstein, "The robot is the tether: active, adaptive power routing for modular robots with unary inter-robot connectors," in Proceedings of the IEEE IRS/RSJ International Conference on Intelligent Robots and Systems (IROS '05), pp. 2960-2967, August 2005.

[97] M. Shimizu, A. Ishiguro, and T. Kawakatsu, "A modular robot that exploits a spontaneous connectivity control mechanism," in Proceedings of the IEEE IRS/RSJ International Conference on Intelligent Robots and Systems (IROS '05), pp. 2658-2663, Edmonton, Canada, August 2005.

[98] M. Shimizu, T. Mori, and A. Ishiguro, "A development of a modular robot that enables adaptive reconfiguration," in Proceedings of the IEEE/RSJ International Conference on Intelligent Robots and Systems (IROS '06), pp. 174-179, Beijing, China, October 2006.

[99] M. E. Karagozler, S. C. Goldstein, and J. R. Reid, "Stressdriven MEMS assembly + electrostatic forces $=1 \mathrm{~mm}$ diameter robot," in Proceedings of the IEEE/RSJ International Conference on Intelligent Robots and Systems (IROS '09), pp. 2763-2769, IEEE, St. Louis, Mo, USA, October 2009.

[100] B. R. Donald, C. G. Levey, C. G. McGray, I. Paprotny, and D. Rus, "An untethered, electrostatic, globally controllable MEMS micro-robot," Journal of Microelectromechanical Systems, vol. 15, no. 1, pp. 1-15, 2006.

[101] B. R. Donald, C. G. Levey, and I. Paprotny, "Planar microassembly by parallel actuation of MEMS microrobots," Journal of Microelectromechanical Systems, vol. 17, no. 4, pp. 789-808, 2008.

[102] K. Kotay, D. Rus, M. Vona, and C. McGray, "The selfreconfiguring robotic molecule," in Proceedings of the IEEE International Conference on Robotics and Automation 1998, pp. 424-431, May 1998.
[103] A. Kawakami, A. Torii, K. Motomura, and S. Hirose, "SMC rover: planetary rover with transformable wheels," in Experimental Robotics VIII, vol. 5 of Springer Tracts in Advanced Robotics, pp. 498-506, Springer, Berlin, Germany, 2003.

[104] M. Delrobaei and K. A. McIsaac, "Design and steering control of a center-articulated mobile robot module," Journal of Robotics, vol. 2011, Article ID 621879, 14 pages, 2011.

[105] A. Kamimura, S. Murata, E. Yoshida, H. Kurokawa, K. Tomita, and S. Kokaji, "Self-reconfigurable modular robot-experiments on reconfiguration and locomotion," in Proceedings of the IEEE/RSJ International Conference on Intelligent Robots and Systems, pp. 606-612, IEEE, Maui, Hawaii, USA, November 2001.

[106] M. Mamei and F. Zambonelli, "Programming modular robots with the TOTA middleware," in Proceedings of the 4th International Conference on Engineering Self-organising Systems (ESOA '06), pp. 99-114, Springer, Hakodate, Japan, 2007, http:// dl.acm.org/citation.cfm?id=1763581.1763591.

[107] W. Hongxing, L. Shiyi, Z. Ying, Y. Liang, and W. Tianmiao, "A middleware based control architecture for modular robot systems," in Proceedings of the IEEE/ASME International Conference on Mechtronic and Embedded Systems and Applications (MESA '08), pp. 327-332, Beijing, China, October 2008. 


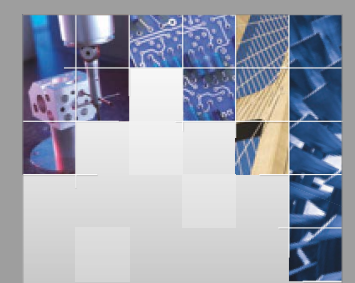

\section{Enfincering}
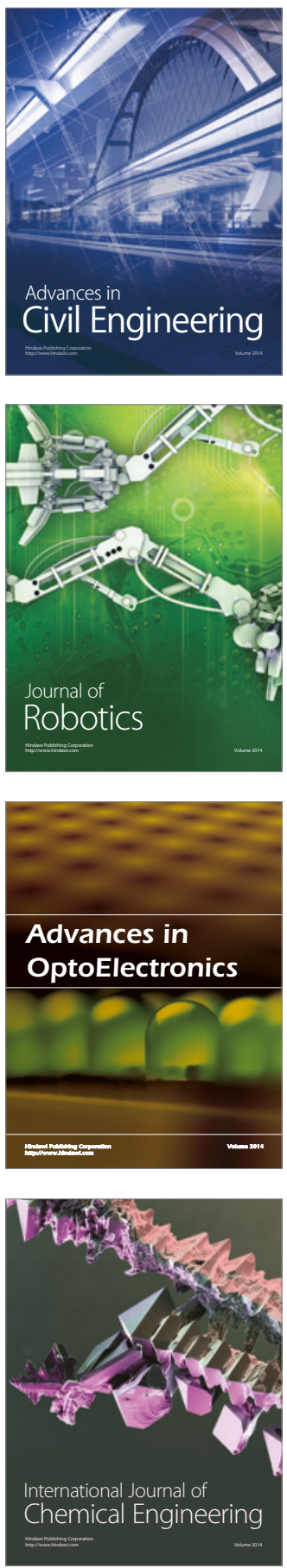

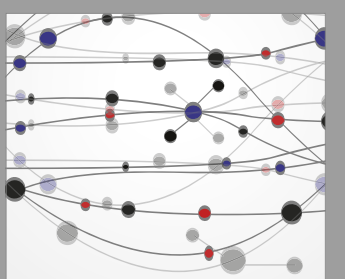

The Scientific World Journal

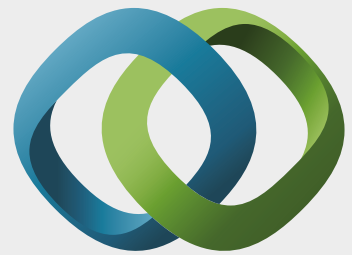

\section{Hindawi}

Submit your manuscripts at

https://www.hindawi.com
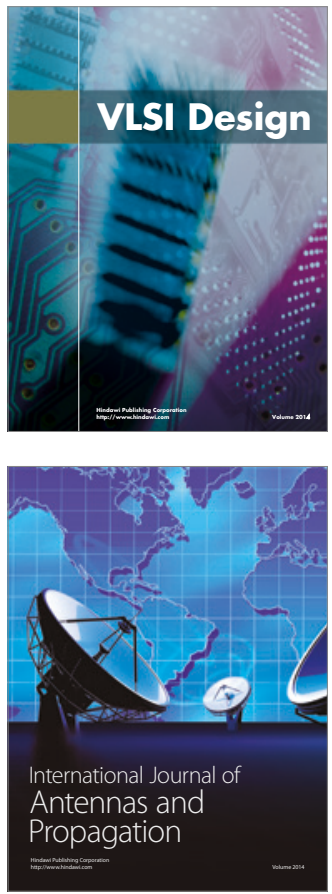

\section{Rotating}

Machinery
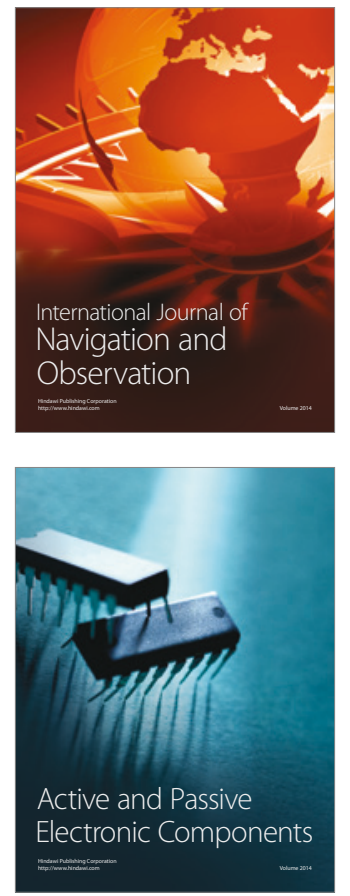
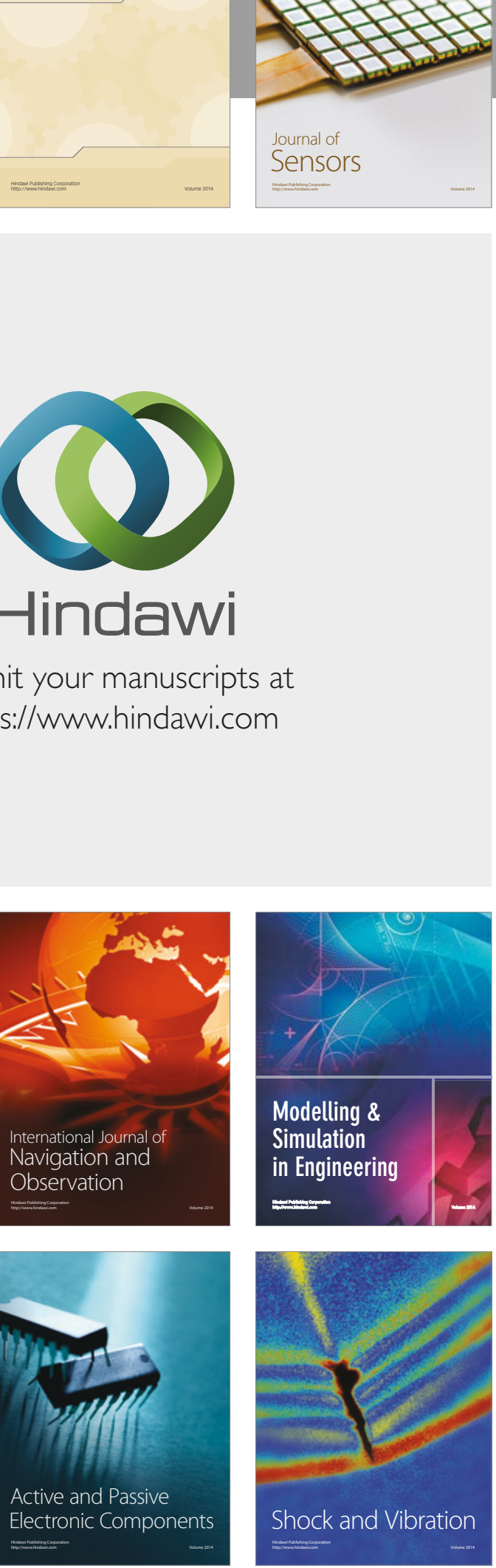
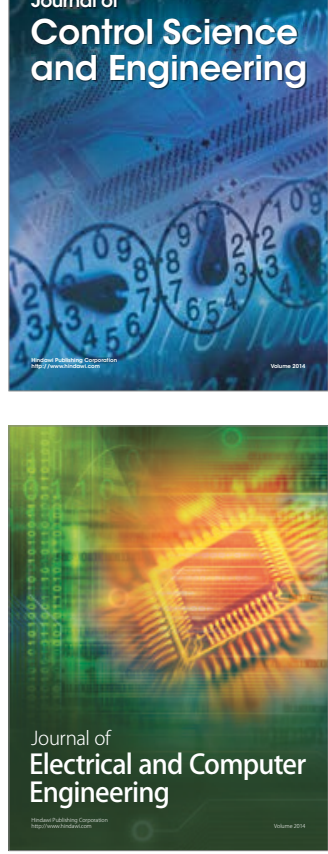

Distributed

Journal of

Control Science

and Engineering
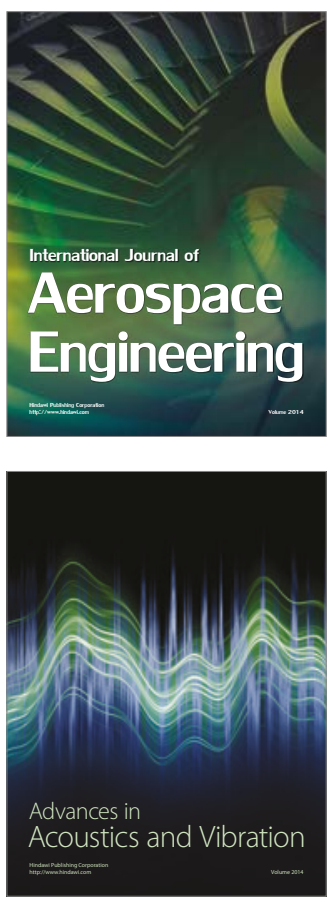

Sensor Networks 